\title{
ON THE SPLINE COLLOCATION METHOD FOR THE SINGLE-LAYER HEAT OPERATOR EQUATION
}

\author{
MARTTI HAMINA AND JUKKA SARANEN
}

\begin{abstract}
We consider a boundary element collocation method for the heat equation. As trial functions we use the tensor products of continuous piecewise linear splines with collocation at the nodal points. Convergence and stability is proved in the case where the spatial domain is a disc. Moreover, practical implementation is discussed in some detail. Numerical experiments confirm our results.
\end{abstract}

\section{INTRODUCTION}

Recently, the Boundary Element Method has been applied to the solution of various time-dependent phenomena such as heat conduction governed by homogeneous parabolic equations $[7,8,15,16,19,20]$ and wave propagation governed by hyperbolic equations [5,6]. The BEM solution of time-dependent problems requires a large computational effort, since results from all previous steps are saved in the computer memory. In addition, careful numerical integration has to be carried out for setting up the matrix equations, which means increasing computing time. Thus, there is a need to reduce the effort, for example by applying simple discretization methods such as collocation or the quadrature method instead of the Galerkin approximation.

In the above-mentioned articles [7, 19] only the Galerkin solution is analyzed. The work [20] of Onishi describes a collocation scheme for the heat equation when the boundary integral equation is of the second kind. On the other hand, there are well-known situations which lead to boundary integral equations of the first kind. In such time-dependent cases no results for the collocation method seem to be available. Therefore, we are inevitably faced with the question of effectively solving such equations. For time-independent problems the collocation, and more recently the quadrature methods, have been analyzed [23, 24].

In this paper we construct and analyze a spline collocation scheme for solving the single-layer heat operator equation, assuming that the spatial domain is two-dimensional and has a smooth boundary. As trial functions we use tensor products of continuous piecewise linear functions with collocation at the nodal points. For the proof of stability and convergence we limit ourselves here to the case of the circle. However, the method can be applied to all smooth

Received by the editor February 27, 1992.

1991 Mathematics Subject Classification. Primary 65N35, 65R20, 45L10, 35K05.

Key words and phrases. Heat equation, collocation, boundary elements.

This work was supported by the Finnish Academy. 
closed curves, although the theory remains to be developed. For our choice of trial functions we are able to establish linear convergence, but it is obvious that faster methods could be defined (by raising the degree of the trial functions) and similarly analyzed. We have carried out some numerical experiments which confirm convergence, indicating even a quadratic rate of convergence. The numerical implementation of the scheme, covering also the general case, will be explained in some detail. Some of our theoretical results were already given in [11].

\section{Single-layer heat OPERATOR EQUATION}

Considering the heat conduction problem, we first introduce two boundary integral approaches for the solution of the homogeneous heat equation with given Dirichlet-type boundary condition and vanishing initial data. For convenience of the reader, we briefly recall some basic situations in which the single-layer heat operator equation arises. For more details see [7, 16].

Let $\Omega \subset \mathbb{R}^{2}$ be a bounded domain with the smooth boundary $\Gamma$. With $0<T<\infty$, we have the heat conduction problem

$$
\begin{cases}-\Delta \Phi+\partial_{t} \Phi=0 & \text { in } \quad Q_{T}=\Omega \times(0, T) \\ \left.\Phi\right|_{\Sigma_{T}}=g & \text { on } \Sigma_{T}=\Gamma \times(0, T) \\ \Phi(\mathbf{x}, 0)=0, & \mathbf{x} \in \Omega\end{cases}
$$

We consider the direct method and the single-layer method for solving the above problem. For this, let

$$
E(\mathbf{x}, t)= \begin{cases}\frac{1}{4 \pi t} \exp \left(-\frac{|\mathbf{x}|^{2}}{4 t}\right), & t>0 \\ 0, & t \leq 0\end{cases}
$$

be the fundamental solution of the two-dimensional heat equation, and let $V$ and $W$ denote the classical single-layer and double-layer heat potentials

$$
(V \sigma)(\mathbf{x}, t)=\int_{0}^{t} \int_{\Gamma} \sigma(\mathbf{y}, \tau) E(\mathbf{x}-\mathbf{y}, t-\tau) d \Gamma_{y} d \tau, \quad(\mathbf{x}, t) \in Q_{T} \cup Q_{T}^{c},
$$

$$
(W \mu)(\mathbf{x}, t)=\int_{0}^{t} \int_{\Gamma} \mu(\mathbf{y}, \tau) \partial_{n_{y}} E(\mathbf{x}-\mathbf{y}, t-\tau) d \Gamma_{y} d \tau, \quad(\mathbf{x}, t) \in Q_{T} \cup Q_{T}^{c},
$$

where $Q_{T}^{c}=\Omega^{c} \times(0, T), \Omega^{c}:=\mathbb{R}^{2} \backslash \bar{\Omega}$ and $\partial_{n_{y}}$ is the exterior normal derivative. For sufficiently smooth functions $\sigma$ and $\mu$ the potentials $V \sigma$ and $W \mu$ have the well-known boundary behavior

$$
\begin{aligned}
\left.V \sigma\right|_{\Sigma_{T}} & =S_{\Gamma} \sigma, \\
\left.W \mu\right|_{\Sigma_{T}} & =\left(D_{\Gamma}-\frac{1}{2} I\right) \mu \quad \text { (interior limit), }
\end{aligned}
$$

where $S_{\Gamma}$ is the single-layer heat operator

$$
\left(S_{\Gamma} \sigma\right)(\mathbf{x}, t)=\int_{0}^{t} \int_{\Gamma} \sigma(\mathbf{y}, \tau) E(\mathbf{x}-\mathbf{y}, t-\tau) d \Gamma_{y} d \tau, \quad \mathbf{x} \in \Gamma,
$$

and $D_{\Gamma}$ is the double-layer heat operator

$$
\left(D_{\Gamma} \mu\right)(\mathbf{x}, t)=\int_{0}^{t} \int_{\Gamma} \mu(\mathbf{y}, \tau) \partial_{n_{y}} E(\mathbf{x}-\mathbf{y}, t-\tau) d \Gamma_{y} d \tau, \quad \mathbf{x} \in \Gamma .
$$


In the direct method the heat potential $\Phi$ in (1.1) is given by means of the representation

$$
\Phi=V \sigma-W g
$$

which by (1.4ii) yields the single-layer heat operator equation

$$
S_{\Gamma} \sigma=\left(\frac{1}{2} I+D_{\Gamma}\right) g
$$

of the first kind. In the indirect single-layer method we use the representation

$$
\Phi=V \tilde{\sigma},
$$

which by (1.4i) implies

$$
S_{\Gamma} \tilde{\sigma}=g .
$$

Thus, both of the above approaches lead to a solution of the single-layer heat operator equation

$$
S_{\Gamma} u_{\Gamma}=g_{\Gamma}
$$

We recall that, in a proper setting of function spaces, the equation (1.11) is uniquely solvable. Moreover, the solutions $\sigma, \tilde{\sigma}$ in (1.8), (1.10) have the interpretations

$$
\begin{gathered}
\sigma=\partial_{n} \Phi^{-}, \\
\tilde{\sigma}=\partial_{n} \Phi^{-}-\partial_{n} \Phi^{+},
\end{gathered}
$$

where + and - denote the exterior and interior limits on the boundary $\Sigma_{T}$.

\section{Preliminaries}

In this section we introduce some notations and define appropriate function spaces which are used for the analysis. Let $\mathbf{x}(\theta), \theta \in \mathbb{R}$, be a smooth 1 periodic parametric representation of the boundary curve $\Gamma$. We assume that the Jacobian is positive, i.e., $\left|\mathbf{x}^{\prime}(\theta)\right|>0$. Denoting $u(\theta, t)=\left|\mathbf{x}^{\prime}(\theta)\right| u_{\Gamma}(\mathbf{x}(\theta), t)$, we obtain

$$
\left(S_{\Gamma} u_{\Gamma}\right)(\mathbf{x}(\theta), t)=(S u)(\theta, t),
$$

where $S$ is the single-layer heat operator

$(S u)(\theta, t)=\int_{0}^{t} \int_{0}^{1} u(\varphi, \tau) E(\mathbf{x}(\theta)-\mathbf{x}(\varphi), t-\tau) d \varphi d \tau, \quad(\theta, t) \in \mathbb{R} \times[0, T]$,

acting in the space of 1-periodic functions with respect to the spatial variable. Thus, the boundary integral equation (1.11) transforms to the integral equation

$$
(S u)(\theta, t)=f(\theta, t), \quad(\theta, t) \in \mathbb{R} \times[0, T],
$$

where $f(\theta, t)=g_{\Gamma}(\mathbf{x}(\theta), t)$.

For numerical purposes we discretize the space-time domain $\mathbb{R} \times[0, T]$. The one-dimensional meshes $0=\theta_{0}<\theta_{1}<\cdots<\theta_{N}=1$ with $h_{\theta}=$ $\max \left(\left|\theta_{n}-\theta_{n-1}\right|\right)$ and $0=t_{0}<t_{1}<\cdots<t_{M}=T$ with $h_{t}=\max \left(\left|t_{m}-t_{m-1}\right|\right)$ induce a rectangular mesh on $\mathbb{R} \times[0, T]$. For this, let $\Delta_{\theta}=\left\{\theta_{n}\right\}_{n \in \mathbb{Z}}$ be the 1 -periodic extension of the mesh $\left\{\theta_{n}\right\}_{n=0}^{N-1}$ to $\mathbb{R}$ and let $\Delta_{t}=\left\{t_{m}\right\}_{m=0}^{M}$. Let $S^{h}$ be the space of continuous piecewise linear 1-periodic splines with respect to 
$\Delta_{\theta}$, and correspondingly let $S_{0, T}^{h}$ be the space of piecewise linear continuous functions $\phi$ such that $\phi(0)=0$. In the space-time domain we use the tensor product spline space $\mathscr{M}:=S^{h} \otimes S_{0, T}^{h}$. Further, we introduce the mean value functionals

$$
(J u)(t):=\int_{0}^{1} u(\theta, t) d \theta, \quad\left(\left(\partial_{t} \otimes J\right) u\right)(t):=\int_{0}^{1} \partial_{t} u(\theta, t) d \theta,
$$

and the trapezoidal rule approximations

$$
\begin{aligned}
\left(J_{\Delta} u\right)(t) & :=\sum_{n=0}^{N-1} \frac{\theta_{n+1}-\theta_{n-1}}{2} u\left(\theta_{n}, t\right), \\
\left(\left(\partial_{t} \otimes J_{\Delta}\right) u\right)(t) & :=\sum_{n=0}^{N-1} \frac{\theta_{n+1}-\theta_{n-1}}{2} \partial_{t} u\left(\theta_{n}, t\right) .
\end{aligned}
$$

The basis of the tensor product space $\mathscr{M}$ is constructed as follows. Let $\left\{\psi_{n} \mid 1 \leq\right.$ $n \leq N\}$ be the familiar Courant basis of $S^{h}$ such that

$$
\psi_{n}\left(\theta_{l}\right)= \begin{cases}1, & l=n+\nu N, \quad \nu \in \mathbb{Z} \\ 0, & l \neq n+\nu N, \quad \nu \in \mathbb{Z}\end{cases}
$$

Then we have

$$
\partial_{\theta} \psi_{n}(\theta)= \begin{cases}\left(\theta_{n}-\theta_{n-1}\right)^{-1}, & \theta_{n+\nu N-1}<\theta<\theta_{n+\nu N}, \nu \in \mathbb{Z}, \\ -\left(\theta_{n+1}-\theta_{n}\right)^{-1}, & \theta_{n+\nu N}<\theta<\theta_{n+\nu N+1}, \nu \in \mathbb{Z}, \\ 0, & \text { elsewhere }\end{cases}
$$

and

$$
J \psi_{n}=\frac{\theta_{n+1}-\theta_{n-1}}{2}, \quad 1 \leq n \leq N .
$$

Correspondingly, we fix the basis functions $\phi_{m}$ of $S_{0, T}^{h}$ by defining

$$
\phi_{m}(t)= \begin{cases}\frac{t}{t_{m}}, & 0 \leq t \leq t_{m}, 1 \leq m \leq M \\ 1, & t_{m} \leq t<T, 1 \leq m \leq M\end{cases}
$$

With $v_{m, n}(\theta, t)=\psi_{n}(\theta) \phi_{m}(t)$, the set $\left\{v_{m, n} \mid 1 \leq n \leq N, 1 \leq m \leq M\right\}$ is a basis of the spline space $\mathscr{M}$, and any function $v \in \mathscr{M}$ has the representation

$$
v(\theta, t)=\sum_{n=1}^{N} \sum_{m=1}^{M} \alpha_{m, n} v_{m, n}(\theta, t) .
$$

For the analysis of the collocation method we need the anisotropic Sobolev spaces. Having introduced the parametric representation for the boundary curve $\Gamma$, it is enough to consider functions which are 1-periodic with respect to the spatial variable. First, for any $r \in \mathbb{R}$, let $H^{r}$ be the Sobolev space of 1-periodic functions on $\mathbb{R}$. The anisotropic spaces

$$
H^{r, s}:=H^{0}\left(\mathbb{R} ; H^{r}\right) \cap H^{s}\left(\mathbb{R} ; H^{0}\right), \quad r, s \in \mathbb{R},
$$

are defined in the usual way $([17$, p. 87$],[18$, p. 8$])$. In terms of the Fourier transform

$$
\left(\mathscr{F}_{t} u\right)(\theta, \tau)=\int_{-\infty}^{\infty} u(\theta, t) e^{-i t \tau} d t
$$


the norm in the space $H^{r, s}$ is given by

$$
\|u\|_{H^{r, s}}^{2}=\int_{-\infty}^{\infty}\left(\left\|\left(\mathscr{F}_{t} u\right)(\cdot, \tau)\right\|_{H^{r}}^{2}+|\tau|^{2 s}\left\|\left(\mathscr{F}_{t} u\right)(\cdot, \tau)\right\|_{H^{0}}^{2}\right) d \tau
$$

The space $H^{r}, s\left(\mathbb{R}_{T}\right), r, s \geq 0,0<T \leq \infty$, is the space of restrictions to $\mathbb{R}_{T}=\mathbb{R} \times(0, T)$ of functions belonging to $H^{r, s}$. Again, we have the Hilbert space

$$
H^{r, s}\left(\mathbb{R}_{T}\right)=H^{0}\left((0, T) ; H^{r}\right) \cap H^{s}\left((0, T) ; H^{0}\right), \quad 0<T \leq \infty,
$$

endowed with the norm

$$
\|u\|_{H^{r, s}\left(\mathbb{R}_{T}\right)}=\left(\int_{0}^{T}\|u(\cdot, t)\|_{H^{r}}^{2} d t+\|u\|_{H^{s}\left((0, T) ; H^{0}\right)}^{2}\right)^{\frac{1}{2}}, \quad 0<T \leq \infty .
$$

We also use equivalent norms defined by

$$
\begin{aligned}
\|u\|_{H^{r, s}\left(\mathbb{R}_{T}\right)}^{2}= & \int_{0}^{T}\|u(\cdot, t)\|_{H^{r}}^{2} d t \\
& +\int_{0}^{T} \int_{0}^{T} \frac{\|u(\cdot, t)-u(\cdot, \tau)\|_{H^{0}}^{2}}{|t-\tau|^{1+2 s}} d t d \tau, \quad 0<s<1,
\end{aligned}
$$

and

$$
\|u\|_{H^{r, s}\left(\mathbf{R}_{T}\right)}^{2}=\int_{0}^{T}\|u(\cdot, t)\|_{H^{r}}^{2} d t+\int_{0}^{1}\|u(\theta, \cdot)\|_{H^{s}(0, T)}^{2} d \theta, \quad 0 \leq s \leq 1 .
$$

Moreover, we introduce the subspace

$$
H_{00}^{r, s}\left(\mathbb{R}_{T}\right)=\left\{u|u=U|_{\mathbb{R}_{T}}: U \in H^{r, s}, U(\cdot, t)=0, t<0\right\}, \quad 0<T \leq \infty .
$$

Thus, for example, $H_{00}^{r, s}\left(\mathbb{R}_{\infty}\right)$ is the space of those functions in $H^{r, s}\left(\mathbb{R}_{\infty}\right)$ for which the zero extension with respect to the time-variable $t$ remains in $H^{r, s}$. Finally, we need the negative-order space $H_{00}^{-r,-s}\left(\mathbb{R}_{T}\right)$ for $0<r<1,0<s<$ $\frac{1}{2}$. These spaces are defined as dual spaces $H_{00}^{-r,-s}\left(\mathbb{R}_{T}\right)=\left(H_{00}^{r, s}\left(\mathbb{R}_{T}\right)\right)^{\prime}$. If there is no danger of confusion, we use the notations

$$
\|\cdot\|=\|\cdot\|_{0,0}, \quad\|\cdot\|_{r, s}=\|\cdot\|_{H^{r, s}\left(\mathbb{R}_{T}\right)} .
$$

An analogous convention applies to the one-dimensional case. In the anisotropic Sobolev spaces the following mapping property is valid [7, 14, 19].

Theorem 2.1. The single-layer heat operator

$$
S: H_{00}^{r, \frac{r}{2}}\left(\mathbb{R}_{T}\right) \rightarrow H_{00}^{r+1, \frac{r+1}{2}}\left(\mathbb{R}_{T}\right)
$$

is an isomorphism for all $r \geq-\frac{1}{2}$. Furthermore, it is coercive such that

$$
(S w \mid w) \geq c\|w\|_{-\frac{1}{2},-\frac{1}{4}}^{2}
$$

for all $w \in H_{00}^{-\frac{1}{2},-\frac{1}{4}}\left(\mathbb{R}_{T}\right)$. Here we assume that $T$ is finite.

In the formula $(2.15)$ the duality pairing extends the $H^{0,0}\left(\mathbb{R}_{T}\right)$ inner product.

\section{ANALYSIS OF THE COLLOCATION EQUATIONS}

We apply piecewise linear approximation both in space and time, together with nodal point collocation. The collocation equations corresponding to the 
equation $S u=f$ are: Find $u_{\Delta} \in \mathscr{M}$ such that

$$
\left(S u_{\Delta}\right)\left(\theta_{n}, t_{m}\right)=f\left(\theta_{n}, t_{m}\right), \quad 1 \leq n \leq N, 1 \leq m \leq M .
$$

We adapt the method developed by Arnold and Wendland [3] for the onedimensional case and extended by Arnold and Saranen [2] for biperiodic problems. In this approach the collocation problem is reduced to a Galerkin problem. In order to establish the required equivalence, we define the bilinear form

$$
\begin{aligned}
B_{\Delta}(w, v)= & \left(\partial_{t} \partial_{\theta} w \mid \partial_{t} \partial_{\theta} v\right)+\left(\left(\partial_{t} \otimes J_{\Delta}\right) w \mid\left(\partial_{t} \otimes J\right) v\right) \\
= & \int_{0}^{1} \int_{0}^{T} \partial_{t} \partial_{\theta} w(\theta, t) \partial_{t} \partial_{\theta} v(\theta, t) d \theta d t \\
& +\int_{0}^{T}\left(\left(\partial_{t} \otimes J_{\Delta}\right) w\right)(t)\left(\left(\partial_{t} \otimes J\right) v\right)(t) d t
\end{aligned}
$$

For the equivalent characterization of the collocation equations (3.1) we need some regularity assumptions on the given right-hand side $f$. Let $\mathscr{C}\left(\overline{\mathbb{R}}_{T}\right)$ be the space of continuous functions $f(\theta, t)$ on the closure $\overline{\mathbb{R}}_{T}$ of $\mathbb{R}_{T}$ such that $f$ is 1 -periodic with respect to the variable $\theta$. Moreover, we define the spaces

$$
\begin{aligned}
& \mathscr{C}_{00}\left(\overline{\mathbb{R}}_{T}\right)=\left\{f \in \mathscr{C}\left(\overline{\mathbb{R}}_{T}\right) \mid f(\theta, 0) \equiv 0\right\}, \\
& \mathscr{H}_{00}^{c}\left(\overline{\mathbb{R}}_{T}\right)=\left\{f \in \mathscr{C}_{00}\left(\overline{\mathbb{R}}_{T}\right) \mid \partial_{\theta} f, \partial_{t} f, \partial_{t} \partial_{\theta} f=\partial_{\theta} \partial_{t} f \in \mathscr{C}\left(\overline{\mathbb{R}}_{T}\right)\right\} .
\end{aligned}
$$

Next we give sufficient conditions on the function $u$ to guarantee the property $S u \in \mathscr{H}_{00}^{c}\left(\overline{\mathbb{R}}_{T}\right)$. Recall that a measurable function $u$ is essentially bounded on $\overline{\mathbb{R}}_{T}$ if there exists $C>0$ such that $|u(\theta, t)| \leq C$ for almost all $(\theta, t) \in \mathbb{R}_{T}$. First we observe the following continuity property.

Lemma 3.1. Assume that $u$ is a measurable and essentially bounded function on $\overline{\mathbb{R}}_{T}$. Then $f=S u \in \mathscr{C}_{00}\left(\overline{\mathbb{R}}_{T}\right)$.

Proof. Let $\left(\theta_{0}, t_{0}\right) \in \mathbb{R}_{T}$. We consider only the case with $0<t_{0}<t$ and $t-t_{0}<\delta \leq \delta_{0},\left|\theta-\theta_{0}\right|<\frac{\delta}{2}$. Introducing the intervals

$$
\begin{gathered}
I\left(\theta_{0}\right)=\left[\theta_{0}-\frac{1}{2}, \theta_{0}+\frac{1}{2}\right], \quad I_{1}\left(\theta_{0}\right)=\left[\theta_{0}-\delta, \theta_{0}+\delta\right], \\
I_{2}\left(\theta_{0}\right)=\left[\theta_{0}-\frac{1}{2}, \theta_{0}-\delta\right] \cup\left[\theta_{0}+\delta, \theta_{0}+\frac{1}{2}\right],
\end{gathered}
$$

we obtain, owing to periodicity with respect to the space variable,

$$
\begin{aligned}
(S u) & (\theta, t)-(S u)\left(\theta_{0}, t_{0}\right) \\
= & \int_{0}^{t_{0}} \int_{I_{2}\left(\theta_{0}\right)} u(\varphi, \tau)\left[E(\mathbf{x}(\theta)-\mathbf{x}(\varphi), t-\tau)-E\left(\mathbf{x}\left(\theta_{0}\right)-\mathbf{x}(\varphi), t_{0}-\tau\right)\right] d \varphi d \tau \\
& +\int_{0}^{t_{0}} \int_{\theta_{0}-\delta}^{\theta_{0}+\delta} u(\varphi, \tau)\left[E(\mathbf{x}(\theta)-\mathbf{x}(\varphi), t-\tau)-E\left(\mathbf{x}\left(\theta_{0}\right)-\mathbf{x}(\varphi), t_{0}-\tau\right)\right] d \varphi d \tau \\
& +\int_{t_{0}}^{t} \int_{\theta_{0}-\frac{1}{2}}^{\theta_{0}+\frac{1}{2}} u(\varphi, \tau) E(\mathbf{x}(\theta)-\mathbf{x}(\varphi), t-\tau) d \varphi d \tau .
\end{aligned}
$$

By using the separation of singularities [10, 22], we have for the kernel the absolutely integrable bounds

$$
\begin{array}{cc}
E(\mathbf{x}(\theta)-\mathbf{x}(\varphi), t-\tau) \leq \frac{C}{(t-\tau)^{\mu}|\mathbf{x}(\theta)-\mathbf{x}(\varphi)|^{2-2 \mu}}, & \frac{1}{2}<\mu<1, \\
E\left(\mathbf{x}\left(\theta_{0}\right)-\mathbf{x}(\varphi), t_{0}-\tau\right) \leq \frac{C}{\left(t_{0}-\tau\right)^{\mu}\left|\mathbf{x}\left(\theta_{0}\right)-\mathbf{x}(\varphi)\right|^{2-2 \mu}}, & \frac{1}{2}<\mu<1 .
\end{array}
$$


The estimates (3.3) together with

$$
c|\theta-\varphi| \leq|\mathbf{x}(\theta)-\mathbf{x}(\varphi)| \leq C|\theta-\varphi|, \quad|\theta-\varphi| \leq 1-\delta_{0},
$$

yield for any positive $\varepsilon$

$$
\begin{aligned}
\left|\int_{0}^{t_{0}} \int_{\theta_{0}-\delta}^{\theta_{0}+\delta} u(\varphi, \tau) E(\mathbf{x}(\theta)-\mathbf{x}(\varphi), t-\tau) d \varphi d \tau\right| & \leq C\|u\|_{\infty} \delta^{2 \mu-1} \leq \varepsilon, \\
\left|\int_{0}^{t_{0}} \int_{\theta_{0}-\delta}^{\theta_{0}+\delta} u(\varphi, \tau) E\left(\mathbf{x}\left(\theta_{0}\right)-\mathbf{x}(\varphi), t_{0}-\tau\right) d \varphi d \tau\right| & \leq C\|u\|_{\infty} \delta^{2 \mu-1} \leq \varepsilon, \\
\left|\int_{t_{0}}^{t} \int_{\theta_{0}-\frac{1}{2}}^{\theta_{0}+\frac{1}{2}} u(\varphi, \tau) E(\mathbf{x}(\theta)-\mathbf{x}(\varphi), t-\tau) d \varphi d \tau\right| & \leq C\|u\|_{\infty}\left(t-t_{0}\right)^{1-\mu} \leq \varepsilon,
\end{aligned}
$$

if $\delta_{0}$ is small enough. Here, $\|u\|_{\infty}$ denotes the essential supremum of $u$ on $\mathbb{R}_{T}$. Moreover, the function $E(\mathbf{x}(\theta)-\mathbf{x}(\varphi), \xi)$ is uniformly continuous in the domain

$$
\left\{\theta|| \theta-\theta_{0} \mid \leq \frac{\delta_{0}}{2}\right\} \times I_{2}\left(\theta_{0}\right) \times[0, T]
$$

and therefore

$$
\int_{0}^{t_{0}} \int_{I_{2}\left(\theta_{0}\right)} u(\varphi, \tau)\left[E(\mathbf{x}(\theta)-\mathbf{x}(\varphi), t-\tau)-E\left(\mathbf{x}\left(\theta_{0}\right)-\mathbf{x}(\varphi), t_{0}-\tau\right)\right] d \varphi d \tau \leq \varepsilon,
$$

if $\delta$ is sufficiently small. The continuity follows from (3.5), (3.6).

Now we introduce the space

$$
\mathscr{C}_{00}^{t}\left(\mathbb{R}_{T}\right)=\{f \mid f(\theta, \cdot) \text { is continuous for almost all } \theta \text { and } f(\theta, 0)=0\} .
$$

The space $\mathscr{L}_{00}\left(\mathbb{R}_{T}\right)$ consists of functions $f \in \mathscr{C}_{00}^{t}\left(\mathbb{R}_{T}\right)$ such that $\partial_{\theta} f \in \mathscr{C}_{00}^{t}\left(\mathbb{R}_{T}\right)$ and the partial derivatives $\partial_{t} f, \partial_{t} \partial_{\theta} f=\partial_{\theta} \partial_{t} f$ are measurable, essentially bounded functions on $\overline{\mathbb{R}}_{T}$. We emphasize that $\mathscr{M}$ is a subspace of $\mathscr{H}_{00}\left(\mathbb{R}_{T}\right)$. The previous lemma is valid for general smooth curves. In the following lemma it is essential that the boundary curve is a circle.

Lemma 3.2. Let $u \in \mathscr{H}_{00}\left(\mathbb{R}_{T}\right)$. Then we have $S u \in \mathscr{H}_{00}^{c}\left(\overline{\mathbb{R}}_{T}\right)$, and the following commutation relations are true:

$$
\begin{aligned}
& \partial_{\theta}(S u)(\theta, t)=\left(S\left(\partial_{\varphi} u\right)\right)(\theta, t), \\
& \partial_{t}(S u)(\theta, t)=\left(S\left(\partial_{\tau} u\right)\right)(\theta, t), \\
& \partial_{t} \partial_{\theta}(S u)(\theta, t)=\left(S\left(\partial_{\tau} \partial_{\varphi} u\right)\right)(\theta, t)=\partial_{\theta} \partial_{t}(S u)(\theta, t), \\
& J(S u)(t)=S(J u)(t), \\
& \left(\left(\partial_{t} \otimes J\right)(S u)\right)(t)=\left(S\left(\partial_{\tau} \otimes J\right) u\right)(t) .
\end{aligned}
$$

Proof. Using the heat kernel corresponding to the circle with radius $r$,

$$
E_{r}(\theta-\varphi, t-\tau)=\frac{\exp \left(-\frac{r^{2} \sin ^{2}(\pi(\theta-\varphi)}{4(t-\tau)}\right)}{4 \pi(t-\tau)}
$$


we have

$$
\begin{aligned}
& \frac{(S u)(\theta+\delta, t)-(S u)(\theta, t)}{\delta} \\
& =\int_{0}^{t} \int_{0}^{1} \frac{u(\varphi+\delta, \tau)-u(\varphi, \tau)}{\delta} E_{r}(\theta-\varphi, t-\tau) d \varphi d \tau \\
& =\int_{0}^{t} \int_{0}^{1}\left(\frac{1}{\delta} \int_{\varphi}^{\varphi+\delta} \partial_{\sigma} u(\sigma, \tau) d \sigma\right) E_{r}(\theta-\varphi, t-\tau) d \varphi d \tau
\end{aligned}
$$

The existence of the partial derivative $\partial_{\varphi} u$ implies that the limit

$$
\lim _{\delta \rightarrow 0} \frac{1}{\delta} \int_{\varphi}^{\varphi+\delta} \partial_{\sigma} u(\sigma, \tau) d \sigma=\lim _{\delta \rightarrow 0} \frac{u(\varphi+\delta, \tau)-u(\varphi, \tau)}{\delta}=\partial_{\varphi} u(\varphi, \tau)
$$

exists for almost all $\varphi \in \mathbb{R}, \tau \in(0, t)$. Furthermore, since $\partial_{\varphi} u$ is essentially bounded, we have

$$
\frac{1}{\delta} \int_{\varphi}^{\varphi+\delta}\left|\partial_{\sigma} u(\sigma, \tau)\right| d \sigma \leq C
$$

and the limit

$$
\lim _{\delta \rightarrow 0} \frac{1}{\delta}[(S u)(\theta+\delta, t)-(S u)(\theta, t)]=\int_{0}^{t} \int_{0}^{1} \partial_{\varphi} u(\varphi, \tau) E_{r}(\theta-\varphi, t-\tau) d \varphi d \tau
$$

exists by the Lebesgue dominated convergence theorem, proving (3.7). Next we consider (3.8). The condition $u(\theta, 0)=0$ yields

$$
\begin{aligned}
\frac{(S u)(\theta, t)-(S u)(\theta, t-\delta)}{\delta} & \\
= & \int_{\delta}^{t} \int_{0}^{1}\left(\frac{1}{\delta} \int_{\tau-\delta}^{\tau} \partial_{\sigma} u(\varphi, \sigma) d \sigma\right) E_{r}(\theta-\varphi, t-\tau) d \varphi d \tau \\
& +\int_{0}^{\delta} \int_{0}^{1}\left(\frac{1}{\delta} \int_{0}^{\tau} \partial_{\sigma} u(\varphi, \sigma) d \sigma\right) E_{r}(\theta-\varphi, t-\tau) d \varphi d \tau
\end{aligned}
$$

Again, the Lebesgue dominated convergence theorem implies the existence of the limit

$$
\lim _{\delta \rightarrow 0} \frac{1}{\delta}[(S u)(\theta, t)-(S u)(\theta, t-\delta)]=\int_{0}^{t} \int_{0}^{1} \partial_{\tau} u(\varphi, \tau) E_{r}(\theta-\varphi, t-\tau) d \varphi d \tau
$$

and (3.8) is proved. Similarly, we get

$$
\left(\partial_{t} \partial_{\theta}(S u)\right)(\theta, t)=\left(\partial_{t}\left(S \partial_{\varphi} u\right)\right)(\theta, t)=\left(S\left(\partial_{\tau} \partial_{\varphi} u\right)\right)(\theta, t)
$$

by using the assumption $\partial_{\varphi} u(\varphi, 0)=0$. On the other hand, we have

$$
\left(\partial_{\theta} \partial_{t}(S u)\right)(\theta, t)=\left(\partial_{\theta}\left(S \partial_{\tau} u\right)\right)(\theta, t)=\left(S\left(\partial_{\varphi} \partial_{\tau} u\right)\right)(\theta, t)=\left(S\left(\partial_{\tau} \partial_{\varphi} u\right)\right)(\theta, t)
$$

where we need only the condition $u(\theta, 0)=0$. To prove (3.10), we consider the mean value $J$ with respect to the spatial variable,

$$
\begin{aligned}
(J(S u))(t) & =\int_{0}^{1} \int_{0}^{t} \int_{0}^{1} \frac{1}{4 \pi} u(\varphi, \tau) \frac{1}{t-\tau} e^{-\frac{1-\cos (2 \pi(\theta-\varphi))}{2(t-\tau)}} d \varphi d \tau d \theta \\
& =\int_{0}^{t} \int_{0}^{1} \frac{1}{4 \pi} u(\varphi, \tau) \frac{1}{t-\tau} e^{-\frac{1}{2(t-\tau)}}\left(\int_{0}^{1} e^{\frac{\cos (2 \pi(\theta-\varphi))}{2(t-\tau)}} d \theta\right) d \varphi d \tau
\end{aligned}
$$


In terms of the modified Bessel function of order zero [1]

$$
I_{0}(z)=\frac{1}{\pi} \int_{0}^{\pi} e^{z \cos (\theta)} d \theta
$$

we have

$$
\int_{0}^{1} e^{\frac{\cos (2 \pi(\theta-\varphi))}{2(t-\tau)}} d \theta=I_{0}\left(\frac{1}{2(t-\tau)}\right) .
$$

Observe that the integral in (3.13) is independent of the variable $\varphi$. Thus, (3.12) becomes

$$
(J(S u))(t)=\int_{0}^{t} \frac{1}{4 \pi} \frac{(J u)(\tau)}{t-\tau} e^{-\frac{1}{2(t-\tau)}} I_{0}\left(\frac{1}{2(t-\tau)}\right) d \tau .
$$

On the other hand, by (3.13),

$$
\begin{aligned}
(S J u)(\theta, t) & =\int_{0}^{t} \int_{0}^{1} \frac{1}{4 \pi}(J u)(\tau) \frac{1}{t-\tau} e^{-\frac{1-\cos (2 \pi(\theta-\varphi))}{2(t-\tau)}} d \varphi d \tau \\
& =\int_{0}^{t} \frac{1}{4 \pi} \frac{(J u)(\tau)}{t-\tau} e^{-\frac{1}{2(t-\tau)}} I_{0}\left(\frac{1}{2(t-\tau)}\right) d \tau=(J(S u))(t)
\end{aligned}
$$

which proves (3.10). Finally, the commutation relation (3.11) follows from (3.8), (3.10). The property $S u \in \mathscr{H}_{00}^{c}\left(\overline{\mathbb{R}}_{T}\right)$ is a consequence of Lemma 3.1 and (3.9).

Theorem 3.1. Let $u \in \mathscr{H}_{00}\left(\mathbb{R}_{T}\right)$ be the solution of the equation $S u=f$. Then the function $u_{\Delta} \in \mathscr{M}$ is a solution of the collocation problem (3.1) if and only if the equations

$$
B_{\Delta}\left(S u_{\Delta}, v\right)=B_{\Delta}(S u, v), \quad v \in \mathscr{M}
$$

are valid.

Proof. We denote $\tilde{w}=w-f$, where $w=S u_{\Delta}$. From (2.6), (2.8) we obtain

$$
\begin{aligned}
\int_{0}^{1} \int_{0}^{T} \partial_{t} \partial_{\theta} \tilde{w}(\theta, t) \partial_{t} \partial_{\theta} v_{m, n}(\theta, t) d \theta d t \\
\quad=\frac{1}{t_{m}} \int_{0}^{1}\left[\int_{0}^{t_{m}} \partial_{t} \partial_{\theta} \tilde{w}(\theta, t) d t\right] \partial_{\theta} \psi_{n}(\theta) d \theta \\
\quad=\frac{1}{t_{m}} \int_{0}^{1}\left[\partial_{\theta} \tilde{w}\left(\theta, t_{m}\right)-\partial_{\theta} \tilde{w}(\theta, 0)\right] \partial_{\theta} \psi_{n}(\theta) d \theta \\
\quad=\frac{1}{t_{m}}\left[\frac{\tilde{w}\left(\theta_{n}, t_{m}\right)-\tilde{w}\left(\theta_{n-1}, t_{m}\right)}{\theta_{n}-\theta_{n-1}}-\frac{\tilde{w}\left(\theta_{n+1}, t_{m}\right)-\tilde{w}\left(\theta_{n}, t_{m}\right)}{\theta_{n+1}-\theta_{n}}\right],
\end{aligned}
$$

where in the last step the property $\tilde{w}(\theta, 0)=0$ was used. Moreover, we have

$$
\left(\left(\partial_{t} \otimes J\right) v_{m, n}\right)(t)= \begin{cases}\frac{J \psi_{n}}{t_{m}}, & 0<t<t_{m}, \\ 0, & t_{m}<t<T,\end{cases}
$$


which by $(2.5)$ yields

$$
\begin{aligned}
& \int_{0}^{T}\left(\left(\partial_{t} \otimes J_{\Delta}\right) \tilde{w}\right)(t)\left(\left(\partial_{t} \otimes J\right) v_{m, n}\right)(t) d t \\
& \quad=\frac{J \psi_{n}}{t_{m}} \sum_{l=0}^{N-1} \frac{\theta_{l+1}-\theta_{l-1}}{2} \int_{0}^{t_{m}} \partial_{t} \tilde{w}\left(\theta_{l}, t\right) d t \\
& \quad=\frac{J \psi_{n}}{t_{m}} \sum_{l=0}^{N-1} \frac{\theta_{l+1}-\theta_{l-1}}{2} \tilde{w}\left(\theta_{l}, t_{m}\right)
\end{aligned}
$$

where we have again used the property $\tilde{w}\left(\theta_{l}, 0\right)=0$.

After these preparations we are able to prove our assertion. Suppose first that $u_{\Delta}$ satisfies the collocation equations, i.e., the nodal values $\tilde{w}\left(\theta_{n}, t_{m}\right)$ vanish. According to (3.2), (2.9), (3.16) and (3.17) the bilinear form $B_{\Delta}(\tilde{w}, v)$ reduces to a linear combination of the nodal values of $\tilde{w}$, and consequently $B_{\Delta}(\tilde{w}, v)=0$ for all $v \in \mathscr{M}$. Conversely, assume that (3.15) is valid. We use the identity

$$
B_{\Delta}(\tilde{w}, J v)=\int_{0}^{T}\left(\left(\partial_{t} \otimes J_{\Delta}\right) \tilde{w}\right)(t)\left(\left(\partial_{t} \otimes J\right) v\right)(t) d t .
$$

For $v \in \mathscr{M}$ we also have $J v \in \mathscr{M}$. Therefore, (3.18) and (3.15) imply

$$
\int_{0}^{T}\left(\left(\partial_{t} \otimes J_{\Delta}\right) \tilde{w}\right)(t)\left(\left(\partial_{t} \otimes J\right) v\right)(t) d t=0, \quad v \in \mathscr{M},
$$

and (3.2) thus yields

$$
\left(\partial_{t} \partial_{\theta} \tilde{w} \mid \partial_{t} \partial_{\theta} v\right)=0, \quad v \in \mathscr{M}
$$

By virtue of (3.20) and (3.16),

$$
\frac{\tilde{w}\left(\theta_{n+1}, t_{m}\right)-\tilde{w}\left(\theta_{n}, t_{m}\right)}{\theta_{n+1}-\theta_{n}}-\frac{\tilde{w}\left(\theta_{n}, t_{m}\right)-\tilde{w}\left(\theta_{n-1}, t_{m}\right)}{\theta_{n}-\theta_{n-1}}=0
$$

for $1 \leq n \leq N, 1 \leq m \leq M$. Let $m$ be fixed. Then we have for all $n=$ $1, \ldots, N$,

$$
\frac{\tilde{w}\left(\theta_{n+1}, t_{m}\right)-\tilde{w}\left(\theta_{n}, t_{m}\right)}{\theta_{n+1}-\theta_{n}}=\frac{\tilde{w}\left(\theta_{n}, t_{m}\right)-\tilde{w}\left(\theta_{n-1}, t_{m}\right)}{\theta_{n}-\theta_{n-1}}=C_{m} .
$$

Using the periodicity of $\tilde{w}$ with respect to $\theta$, we obtain

$$
\sum_{n=1}^{N}\left(\tilde{w}\left(\theta_{n}, t_{m}\right)-\tilde{w}\left(\theta_{n-1}, t_{m}\right)\right)=\tilde{w}\left(\theta_{N}, t_{m}\right)-\tilde{w}\left(\theta_{0}, t_{m}\right)=0 .
$$

On the other hand, (3.22) implies

$$
\sum_{n=1}^{N}\left(\tilde{w}\left(\theta_{n}, t_{m}\right)-\tilde{w}\left(\theta_{n-1}, t_{m}\right)\right)=C_{m} \sum_{n=1}^{N}\left(\theta_{n}-\theta_{n-1}\right)=C_{m},
$$

which, with (3.23), gives $C_{m}=0$. Inserting this back into (3.22), we have

$$
\tilde{w}\left(\theta_{n}, t_{m}\right)=\tilde{w}\left(\theta_{0}, t_{m}\right), \quad 1 \leq n \leq N .
$$


Finally, according to (3.17), (3.19), (3.25),

$$
0=\frac{J \psi_{n}}{t_{m}} \sum_{l=0}^{N-1} \frac{\theta_{l+1}-\theta_{l-1}}{2} \tilde{w}\left(\theta_{l}, t_{m}\right)=\frac{J \psi_{n}}{t_{m}} \tilde{w}\left(\theta_{0}, t_{m}\right) .
$$

Since $J \psi_{n}$ is nonzero, the value $\tilde{w}\left(\theta_{0}, t_{m}\right)$ vanishes and, in view of the formula (3.25), all nodal values $\tilde{w}\left(\theta_{n}, t_{m}\right)$ vanish. Thus, $u_{\Delta}$ satisfies the collocation equations (3.1).

\section{Stability analysis}

Here we prove the unique solvability of the collocation equations (3.1) if the spatial discretization parameter $h_{\theta}$ is small enough. This result is a consequence of a coercivity estimate for the bilinear form $B_{\Delta}(S u, v)$, which yields stability and convergence of the method. To prove the coercivity, we consider the form $B_{\Delta}(w, v)$ as a small perturbation of the bilinear form

$$
B(w, v)=\left(\partial_{t} \partial_{\theta} w \mid \partial_{t} \partial_{\theta} v\right)+\left(\left(\partial_{t} \otimes J\right) w \mid\left(\partial_{t} \otimes J\right) v\right) .
$$

For brevity, we introduce the notation

$$
\|\| v||_{-\frac{1}{2},-\frac{1}{4}}=\left\|\partial_{t} \partial_{\theta} v\right\|_{-\frac{1}{2},-\frac{1}{4}}+\left\|\left(\partial_{t} \otimes J\right) v\right\|_{-\frac{1}{2},-\frac{1}{4}} .
$$

In any space of functions where this expression is well defined and finite, it gives a seminorm. It is essential to see that $\left.|| \cdot||\right|_{-\frac{1}{2},-\frac{1}{4}}$ is a norm in the subspace $\mathscr{M}$. This is proved in the following lemma.

Lemma 4.1. The mapping $v \mapsto\|v\|_{-\frac{1}{2},-\frac{1}{4}}$ defines a norm in the space $\mathscr{M}=$ $S^{h} \otimes S_{0, T}^{h}$.

Proof. It suffices to show that the condition

$$
\|\mid v\|_{-\frac{1}{2},-\frac{1}{4}}=0 \text { for } v \in \mathscr{M}
$$

implies $v(\theta, t) \equiv 0$. Now, assuming (4.3), we obtain from the representation (2.9)

$$
\left(\partial_{t} \partial_{\theta} v\right)(\theta, t)=\partial_{t}\left(\sum_{m=1}^{M} \sum_{n=1}^{N} \alpha_{m, n}\left(\partial_{\theta} \psi_{n}\right)(\theta) \phi_{m}(t)\right)
$$

and

$$
\left(\left(\partial_{t} \otimes J\right) v\right)(t)=\partial_{t}\left(\sum_{m=1}^{M} \sum_{n=1}^{N} \alpha_{m, n}\left(J \psi_{n}\right) \phi_{m}(t)\right) .
$$

According to (4.3), (4.4) we have

$$
\sum_{m=1}^{M}\left(\sum_{n=1}^{N} \alpha_{m, n}\left(\partial_{\theta} \psi_{n}\right)(\theta)\right) \phi_{m}(t) \equiv C(\theta) .
$$

In fact, $C(\theta)=0$, since $\phi_{m}(0)=0$ for all $m=1, \ldots, M$. This yields

$$
\sum_{n=1}^{N} \alpha_{m, n}\left(\partial_{\theta} \psi_{n}\right)(\theta) \equiv 0, \quad m=1, \ldots, M
$$


Analogously, from (4.3), (4.5) we deduce that

$$
\sum_{n=1}^{N} \alpha_{m, n}\left(J \psi_{n}\right)=0, \quad m=1, \ldots, M .
$$

Adding (4.7) and (4.8) together gives

$$
\sum_{n=1}^{N} \alpha_{m, n}\left(\partial_{\theta}+J\right) \psi_{n}=\left(\partial_{\theta}+J\right)\left(\sum_{n=1}^{N} \alpha_{m, n} \psi_{n}\right) \equiv 0, \quad m=1, \ldots, M .
$$

The operator $\left(\partial_{\theta}+J\right)$ is an isomorphism from $S^{h}$ to the space of piecewise constant periodic splines. Thus,

$$
\sum_{n=1}^{N} \alpha_{m, n} \psi_{n}=0, \quad m=1, \ldots, M,
$$

which implies that all the coefficients $\alpha_{m, n}$ vanish, proving our statement.

Our next aim is to establish the required continuity and coercivity of the form $B_{\Delta}(S u, v)$. These properties are valid in the space $\mathscr{H}_{00}\left(\mathbb{R}_{T}\right)$. First we consider the form $B(S u, v)$.

Lemma 4.2. We have the continuity estimate

$$
|B(S u, v)| \leq\left.\left. c|||u|\right|_{-\frac{1}{2},-\frac{1}{4}}|| v||\right|_{-\frac{1}{2},-\frac{1}{4}}
$$

for all $u, v \in \mathscr{H}_{00}\left(\mathbb{R}_{T}\right)$ and the coercivity

$$
B(S v, v) \geq c\|\| v \|_{-\frac{1}{2},-\frac{1}{4}}^{2}, \quad v \in \mathscr{H}_{00}\left(\mathbb{R}_{T}\right) .
$$

Proof. We have by the commutation relations (3.9), (3.11)

$$
B(S u, v)=\left(S \partial_{t} \partial_{\theta} u \mid \partial_{t} \partial_{\theta} v\right)+\left(S\left(\partial_{t} \otimes J\right) u \mid\left(\partial_{t} \otimes J\right) v\right) .
$$

The mapping property (2.14) gives

$$
\begin{aligned}
& |B(S u, v)| \leq\left\|S \partial_{t} \partial_{\theta} u\right\|_{\frac{1}{2}, \frac{1}{4}}\left\|\partial_{t} \partial_{\theta} v\right\|_{-\frac{1}{2},-\frac{1}{4}}+\left\|S\left(\partial_{t} \otimes J\right) v\right\|_{\frac{1}{2}, \frac{1}{4}}\left\|\left(\partial_{t} \otimes J\right) v\right\|_{-\frac{1}{2},-\frac{1}{4}} \\
& \quad \leq c\left(\left\|\partial_{t} \partial_{\theta} u\right\|_{-\frac{1}{2},-\frac{1}{4}}\left\|\partial_{t} \partial_{\theta} v\right\|_{-\frac{1}{2},-\frac{1}{4}}+\left\|\left(\partial_{t} \otimes J\right) u\right\|_{-\frac{1}{2},-\frac{1}{4}}\left\|\left(\partial_{t} \otimes J\right) v\right\|_{-\frac{1}{2},-\frac{1}{4}}\right) \\
& \quad \leq\left.\left. c\|u\|\right|_{-\frac{1}{2},-\frac{1}{4}}\|v\|\right|_{-\frac{1}{2},-\frac{1}{4}},
\end{aligned}
$$

which proves (4.9). From (4.11), (2.15) we have

$$
\begin{aligned}
B(S v, v) & =\left(S \partial_{t} \partial_{\theta} v \mid \partial_{t} \partial_{\theta} v\right)+\left(S\left(\partial_{t} \otimes J\right) v \mid\left(\partial_{t} \otimes J\right) v\right) \\
& \geq c\left(\left\|\partial_{t} \partial_{\theta} v\right\|_{-\frac{1}{2},-\frac{1}{4}}^{2}+\left\|\left(\partial_{t} \otimes J\right) v\right\|_{-\frac{1}{2},-\frac{1}{4}}^{2}\right) \\
& \geq c\|\| v \|\left.\right|_{-\frac{1}{2},-\frac{1}{4}} ^{2},
\end{aligned}
$$

which yields $(4.10)$.

Concerning the difference $B-B_{\Delta}$, we have the following lemma. 
Lemma 4.3. For all $u, v \in \mathscr{H}_{00}\left(\mathbb{R}_{T}\right)$ there holds

$$
\left|B(S u, v)-B_{\Delta}(S u, v)\right| \leq\left. c h_{\theta}|| u||\right|_{-\frac{1}{2},-\frac{1}{4}}|| v \mid \|_{-\frac{1}{2},-\frac{1}{4}} .
$$

Proof. Using the commutation relations

$$
\left(\partial_{t} \otimes J\right) S u=J S \partial_{t} u, \quad\left(\partial_{t} \otimes J_{\Delta}\right) S u=J_{\Delta} S \partial_{t} u,
$$

which follow from $(2.4),(2.5),(3.8)$, we obtain

$$
\begin{aligned}
\left|B(S u, v)-B_{\Delta}(S u, v)\right| & =\left|\left(\partial_{t} \otimes\left(J-J_{\Delta}\right)(S u) \mid\left(\partial_{t} \otimes J\right) v\right)\right| \\
& =\left|\left(\left(J-J_{\Delta}\right)\left(S \partial_{t} u\right) \mid\left(\partial_{t} \otimes J\right) v\right)\right| \\
& \leq\left\|\left(J-J_{\Delta}\right)\left(S \partial_{t} u\right)\right\|_{\frac{1}{2}, \frac{1}{4}} \mid\left(\partial_{t} \otimes J\right) v \|_{-\frac{1}{2},-\frac{1}{4}} .
\end{aligned}
$$

Define $w=S \partial_{t} u$. Since $\left(J-J_{\Delta}\right) w(\cdot, t)$ is independent of the variable $\theta$, we have by the accuracy of the trapezoidal rule

$$
\left\|\left(J-J_{\Delta}\right) w(\cdot, t)\right\|=\left|\left(J-J_{\Delta}\right) w(\cdot, t)\right| \leq c h_{\theta}\|w(\cdot, t)\|_{H^{1}} .
$$

According to (3.7), (3.10), and (2.14),

$$
\begin{aligned}
\left\|\partial_{\theta} w\right\|_{\frac{1}{2}, \frac{1}{4}} & =\left\|\partial_{\theta} S \partial_{t} u\right\|_{\frac{1}{2}, \frac{1}{4}}=\left\|S \partial_{\theta} \partial_{t} u\right\|_{\frac{1}{2}, \frac{1}{4}} \leq c\left\|\partial_{\theta} \partial_{t} u\right\|_{-\frac{1}{2},-\frac{1}{4}}, \\
\|J w\|_{\frac{1}{2}, \frac{1}{4}} & =\left\|J S \partial_{t} u\right\|_{\frac{1}{2}, \frac{1}{4}}=\left\|S J \partial_{t} u\right\|_{\frac{1}{2}, \frac{1}{4}} \leq c\left\|\left(\partial_{t} \otimes J\right) u\right\|_{-\frac{1}{2},-\frac{1}{4}}
\end{aligned}
$$

The norm (2.12) together with (4.14) yields

$$
\begin{aligned}
\|(J & \left.-J_{\Delta}\right) w \|_{\frac{1}{2}, \frac{1}{4}}^{2} \\
\leq & c \int_{0}^{T}\left|\left(J-J_{\Delta}\right) w(\cdot, t)\right|^{2} d t \\
& +c \int_{0}^{T} \int_{0}^{T} \frac{\left|\left(J-J_{\Delta}\right) w(\cdot, t)-\left(J-J_{\Delta}\right) w\left(\cdot, t^{\prime}\right)\right|^{2}}{\left|t-t^{\prime}\right|^{\frac{3}{2}}} d t d t^{\prime} \\
\leq & c h_{\theta}^{2} \int_{0}^{T}\|w(\cdot, t)\|_{H^{1}}^{2} d t+c h_{\theta}^{2} \int_{0}^{T} \int_{0}^{T} \frac{\left\|w(\cdot, t)-w\left(\cdot, t^{\prime}\right)\right\|_{H^{1}}^{2}}{\left|t-t^{\prime}\right|^{\frac{3}{2}}} d t d t^{\prime} \\
\leq & c h_{\theta}^{2} \int_{0}^{T}\left(\left\|\partial_{\theta} w(\cdot, t)\right\|_{H^{0}}^{2}+|J w(t)|^{2}\right) d t \\
& +c h_{\theta}^{2} \int_{0}^{T} \int_{0}^{T}\left(\frac{\left\|\partial_{\theta} w(\cdot, t)-\partial_{\theta} w\left(\cdot, t^{\prime}\right)\right\|_{H^{0}}^{2}}{\left|t-t^{\prime}\right|^{\frac{3}{2}}}+\frac{\left|(J w)(t)-(J w)\left(t^{\prime}\right)\right|^{2}}{\left|t-t^{\prime}\right|^{\frac{3}{2}}}\right) d t d t^{\prime} \\
\leq & c h_{\theta}^{2}\left(\left\|\partial_{\theta} w\right\|_{\frac{1}{2}, \frac{1}{4}}^{2}+\|J w\|_{\frac{1}{2}, \frac{1}{4}}^{2}\right)
\end{aligned}
$$

and hence, by (4.15),

$$
\left\|\left(J-J_{\Delta}\right) S \partial_{t} u\right\|_{\frac{1}{2}, \frac{1}{4}} \leq c h_{\theta}\|u\| \|_{-\frac{1}{2},-\frac{1}{4}} .
$$

The required estimate (4.12) follows from (4.13) and (4.16).

Combining Lemmas 4.2 and 4.3, we obtain

Lemma 4.4. For all $u, v \in \mathscr{H}_{00}\left(\mathbb{R}_{T}\right)$ we have the continuity estimate

$$
\left|B_{\Delta}(S u, v)\right| \leq c_{1}|||u| \|\left._{-\frac{1}{2},-\frac{1}{4}}|| v||\right|_{-\frac{1}{2},-\frac{1}{4}} .
$$

Moreover, there exists a constant $h_{0}$ such that for all $0<h_{\theta} \leq h_{0}$ there holds

$$
B_{\Delta}(S v, v) \geq c_{2} \mid\|v\|_{-\frac{1}{2},-\frac{1}{4}}^{2}, \quad v \in \mathscr{H}_{00}\left(\mathbb{R}_{T}\right) .
$$

We can now state the main result of this section. 
Theorem 4.1. Assume that the solution $u$ of the equation (2.3) satisfies $u \in$ $\mathscr{H}_{00}\left(\mathbb{R}_{T}\right)$. Then, for all $0<h_{\theta} \leq h_{0}$, there exists a unique solution $u_{\Delta}$ of the collocation equations (3.1). Moreover, we have the stability

$$
\left|\left\|u_{\Delta}\left|\left\|_{-\frac{1}{2},-\frac{1}{4}} \leq \frac{c_{1}}{c_{2}}\right\|\right| u||_{-\frac{1}{2},-\frac{1}{4}}\right.\right.
$$

and the quasi-optimal approximation result

$$
\left\|u-u_{\Delta} \mid\right\|_{-\frac{1}{2},-\frac{1}{4}} \leq\left(1+\frac{c_{1}}{c_{2}}\right) \inf _{v \in \mathscr{M}}\|u-v\|_{-\frac{1}{2},-\frac{1}{4}} .
$$

Proof. For $u \in \mathscr{H}_{00}\left(\mathbb{R}_{T}\right)$ the collocation problem (3.1) is, by Theorem 3.1, equivalent to the equations

$$
B_{\Delta}\left(S u_{\Delta}, v\right)=B_{\Delta}(S u, v), \quad v \in \mathscr{M} .
$$

If $0<h_{\theta} \leq h_{0}$, the existence of a unique solution for the finite-dimensional problem (4.21) follows from the coercivity (4.18), since $\|v \mid\|_{-\frac{1}{2},-\frac{1}{4}}$ is a norm in the subspace $\mathscr{M}$. By (4.18), (4.21), (4.17) there holds

$$
c_{2}|| u_{\Delta}\left|\left\|_{-\frac{1}{2},-\frac{1}{4}}^{2} \leq B_{\Delta}\left(S u_{\Delta}, u_{\Delta}\right)=B_{\Delta}\left(S u, u_{\Delta}\right) \leq c_{1}|||u|||_{-\frac{1}{2},-\frac{1}{4}}|| u_{\Delta}|\||_{-\frac{1}{2},-\frac{1}{4}},\right.\right.
$$

which yields the stability result (4.19). Now (4.20) follows, since

$$
\begin{aligned}
\| u-u_{\Delta}||_{-\frac{1}{2},-\frac{1}{4}} & \leq\left.\left\|u-v||_{-\frac{1}{2},-\frac{1}{4}}+\right\|\left|v-u_{\Delta}\right|\right|_{-\frac{1}{2},-\frac{1}{4}} \\
& \leq\left.\left(1+\frac{c_{1}}{c_{2}}\right)|\| u-v|\right|_{-\frac{1}{2},-\frac{1}{4}}
\end{aligned}
$$

for all $v \in \mathscr{M}$.

\section{Convergence analysis}

In this section we analyze the order of convergence of the collocation approximation (3.1). Based on the quasi-optimal error estimate (4.20), we first discuss the asymptotic accuracy of the approximation when the error is measured by means of the norm $\|\left.|\cdot|\right|_{-\frac{1}{2},-\frac{1}{4}}$. For this, we need error estimates for the $L^{2}$ orthogonal projection $P_{\theta, t}:=P_{\theta} \otimes P_{t}=P_{t} \otimes P_{\theta}: L^{2}\left(\mathbb{R}_{T}\right) \rightarrow \mathscr{M}$. Here, $P_{\theta}$ and $P_{t}$ are the one-dimensional $L^{2}$ orthogonal projections such that $P_{\theta}: H^{0} \rightarrow S^{h}$ and $P_{t}: H^{0}(0, T) \rightarrow S_{0, T}^{h}$. From now on, we require that the meshes $\Delta_{\theta}$ and $\Delta_{t}$ are quasi-uniform. Under this assumption the 1-dimensional inverse estimates

$$
\|\psi\|_{r} \leq c h_{\theta}^{-(r-s)}\|\psi\|_{s}, \quad \psi \in S^{h}, s \leq r \leq 1,
$$

and

$$
\|\phi\|_{r} \leq c h_{t}^{-(r-s)}\|\phi\|_{s}, \quad \phi \in S_{0, T}^{h}, \quad s \leq r \leq 1,
$$

are available [4]. Moreover, the projection operator $P_{\theta}$ satisfies the approximation result [9]

$$
\left\|u-P_{\theta} u\right\|_{H^{r}} \leq c h_{\theta}^{s-r}\|u\|_{H^{s}}, \quad u \in H^{s},
$$

$0 \leq r \leq s \leq 2, r<\frac{3}{2}$. In the case of the projection $P_{t}$ we confine ourselves to the (easily verified) property

$$
\left\|u-P_{t} u\right\|_{H^{r}(0, T)} \leq c h_{t}^{s-r}\|u\|_{H^{s}(0, T)}, \quad u \in H^{s}(0, T),
$$


where $0 \leq r \leq 1, s$ takes the values $s=1,2$, and $u \in H^{s}(0, T), u(0)=0$. In the following proof we use the further estimates

$$
\left\|\partial_{\theta} u\right\|_{-r, 0} \leq c\|u\|_{1-r, 0}, \quad u \in H^{1-r, 0}\left(\mathbb{R}_{T}\right), 0 \leq r<1,
$$

and

$$
\left\|\partial_{t} u\right\|_{0,-s} \leq c\|u\|_{0,1-s}, \quad u \in H^{0,1-s}\left(\mathbb{R}_{T}\right), 0 \leq s \leq \frac{1}{2} .
$$

These results can be verified using the eigenfunction representation of the anisotropic norms, as described, e.g., in [17, pp. 86-88].

For the proof of our convergence results we make additional regularity assumptions on the solution $u$ of (2.3). It is obvious that these conditions can be weakened in many ways, but we prefer to keep the presentation short and elementary.

Lemma 5.1. Assume that $u \in \mathscr{H}_{00}\left(\mathbb{R}_{T}\right)$ such that $u(\theta, \cdot),\left(\partial_{\theta} u\right)(\theta, \cdot) \in H^{2}(0, T)$ for almost all $\theta$, and $\left(\partial_{t} u\right)(\cdot, t) \in H^{2}$ for almost all $t$. Then we have the approximation result

(5.7) $\quad\left\|u-P_{\theta, t} u\right\|\left\|_{-\frac{1}{2},-\frac{1}{4}} \leq c h_{\theta}^{\frac{3}{2}}\right\| \partial_{t} u \|_{2,0}+c h_{t}\left(h_{\theta}^{\frac{1}{2}}+h_{t}^{\frac{1}{4}}\right)\left(\|u\|_{0,2}+\left\|\partial_{\theta} u\right\|_{0,2}\right)$.

Proof. We use the decomposition

(5.8) $\partial_{\theta} \partial_{t}\left(u-P_{\theta, t} u\right)=\partial_{\theta}\left(I-P_{\theta}\right) \partial_{t} u+\partial_{t}\left(I-P_{t}\right) \partial_{\theta} u-\partial_{\theta}\left(I-P_{\theta}\right) \partial_{t}\left(I-P_{t}\right) u$.

By (5.5), (5.3) there holds

$$
\begin{aligned}
\left\|\partial_{\theta}\left(I-P_{\theta}\right) \partial_{t} u\right\|_{-\frac{1}{2},-\frac{1}{4}}^{2} & \leq\left\|\partial_{\theta}\left(I-P_{\theta}\right) \partial_{t} u\right\|_{-\frac{1}{2}, 0}^{2} \leq c\left\|\left(I-P_{\theta}\right) \partial_{t} u\right\|_{\frac{1}{2}, 0}^{2} \\
& \leq c \int_{0}^{T}\left\|\left(I-P_{\theta}\right) \partial_{t} u(\cdot, t)\right\|_{H^{\frac{1}{2}}}^{2} d t \\
& \leq c h_{\theta}^{2\left(r-\frac{1}{2}\right)} \int_{0}^{T}\left\|\partial_{t} u(\cdot, t)\right\|_{H^{r}}^{2} d t, \quad r=1,2,
\end{aligned}
$$

which yields

$$
\left\|\partial_{\theta}\left(I-P_{\theta}\right) \partial_{t} u\right\|_{-\frac{1}{2},-\frac{1}{4}} \leq c h_{\theta}^{r-\frac{1}{2}}\left\|\partial_{t} u\right\|_{r, 0}, \quad r=1,2 .
$$

Analogously, by (5.6), (5.4),

$$
\begin{array}{r}
\left\|\partial_{t}\left(I-P_{t}\right) \partial_{\theta} u\right\|_{-\frac{1}{2},-\frac{1}{4}}^{2} \leq\left\|\partial_{t}\left(I-P_{t}\right) \partial_{\theta} u\right\|_{0,-\frac{1}{4}}^{2} \leq c\left\|\left(I-P_{t}\right) \partial_{\theta} u\right\|_{0, \frac{3}{4}}^{2} \\
\leq c \int_{0}^{1}\left\|\left(I-P_{t}\right) \partial_{\theta} u(\theta, \cdot)\right\|_{H^{\frac{3}{4}(0, T)}}^{2} d \theta \leq c h_{t}^{\frac{5}{2}}\left\|\partial_{\theta} u\right\|_{0,2}^{2} .
\end{array}
$$

For the last term in (5.8) we first use (5.9),

$$
\begin{aligned}
& \left\|\partial_{\theta}\left(I-P_{\theta}\right) \partial_{t}\left(I-P_{t}\right) u\right\|_{-\frac{1}{2},-\frac{1}{4}}^{2} \leq c h_{\theta}\left\|\partial_{t}\left(I-P_{t}\right) u\right\|_{1,0}^{2} \\
& \quad \leq c h_{\theta} \int_{0}^{1}\left(\left\|\partial_{t}\left(I-P_{t}\right) \partial_{\theta} u(\theta, \cdot)\right\|_{H^{0}(0, T)}^{2}+\left\|\partial_{t}\left(I-P_{t}\right) u(\theta, \cdot)\right\|_{H^{0}(0, T)}^{2}\right) d \theta \\
& \quad \leq c h_{\theta} h_{t}^{2}\left(\|u\|_{0,2}^{2}+\left\|\partial_{\theta} u\right\|_{0,2}^{2}\right) .
\end{aligned}
$$

This, with (5.8), (5.9), and (5.10), gives the estimate

$$
\begin{aligned}
\left\|\partial_{\theta} \partial_{t}\left(u-P_{\theta, t} u\right)\right\|_{-\frac{1}{2},-\frac{1}{4}} \leq & c h_{\theta}^{\frac{3}{2}}\left\|\partial_{t} u\right\|_{2,0}+c h_{t}^{\frac{5}{4}}\left\|\partial_{\theta} u\right\|_{0,2} \\
& +c h_{\theta}^{\frac{1}{2}} h_{t}\left(\|u\|_{0,2}+\left\|\partial_{\theta} u\right\|_{0,2}\right) .
\end{aligned}
$$


Slightly modifying the proof of $(5.11)$, we get

$$
\left\|\left(\partial_{t} \otimes J\right)\left(u-P_{\theta, t} u\right)\right\|_{-\frac{1}{2},-\frac{1}{4}} \leq c h_{t}^{\frac{5}{4}}\|u\|_{0,2},
$$

and (5.7) follows by (5.11), (5.12).

From Theorem 4.1 and Lemma 5.1 we obtain

Theorem 5.1. Assume that the solution $u$ of the equation $S u=f$ satisfies the assumptions $u \in \mathscr{H}_{00}\left(\mathbb{R}_{T}\right)$ such that $u(\theta, \cdot),\left(\partial_{\theta} u\right)(\theta, \cdot) \in H^{2}(0, T)$ for almost all $\theta$, and $\left(\partial_{t} u\right)(\cdot, t) \in H^{2}$ for almost all $t$. Then the collocation approximation $u_{\Delta} \in \mathscr{M}$ defined by (3.1) furnishes the asymptotic error estimate

$$
\left\|u-u_{\Delta} \mid\right\|_{-\frac{1}{2},-\frac{1}{4}} \leq c h_{\theta}^{\frac{3}{2}}\left\|\partial_{t} u\right\|_{2,0}+c h_{t}\left(h_{\theta}^{\frac{1}{2}}+h_{t}^{\frac{1}{4}}\right)\left(\|u\|_{0,2}+\left\|\partial_{\theta} u\right\|_{0,2}\right) .
$$

By the estimate (5.13) the time step $h_{t}$ dominates the order of convergence. This effect can be compensated by letting the time-discretization be finer than the discretization in the space variable.

Theorem 5.2. Let the assumptions of Theorem 5.1 be valid. Moreover, suppose that $h_{\theta} \leq h$ and $h_{t} \leq c h^{\nu}$, where $h \leq h_{0}$ is sufficiently small. Then the collocation approximation $u_{\Delta}$ satisfies

$$
\left.\left\|u-u_{\Delta}\right\|\right|_{-\frac{1}{2},-\frac{1}{4}} \leq \operatorname{ch}^{\frac{3}{2}}\left\|\partial_{t} u\right\|_{2,0}+\operatorname{ch}^{\min \left(\frac{5}{4} \nu, \nu+\frac{1}{2}\right)}\left(\|u\|_{0,2}+\left\|\partial_{\theta} u\right\|_{0,2}\right)
$$

and in particular for $\nu \geq \frac{6}{5}$,

$$
\left\|u-u_{\Delta}\right\|_{-\frac{1}{2},-\frac{1}{4}} \leq \operatorname{ch}^{\frac{3}{2}}\left(\|u\|_{0,2}+\left\|\partial_{\theta} u\right\|_{0,2}+\left\|\partial_{t} u\right\|_{2,0}\right) .
$$

The final aim of this section is to establish pointwise and $L^{2}$ convergence results for the collocation solution. For this, we need the following inverse estimate.

Lemma 5.2. In the space $\mathscr{M}$ we have

$$
\left\|\partial_{t} \partial_{\theta} v\right\|_{0,0}+\left\|\left(\partial_{t} \otimes J\right) v\right\|_{0,0} \leq c \max \left(h_{\theta}^{-\frac{1}{2}}, h_{t}^{-\frac{1}{4}}\right)\|v v\|_{-\frac{1}{2},-\frac{1}{4}} .
$$

Proof. We prove the estimates

$$
\begin{aligned}
\|v\|_{0,0} \leq c \max \left(h_{\theta}^{-r}, h_{t}^{-\frac{r}{2}}\right)\|v\|_{-r,-\frac{r}{2}}, & 0 \leq r<1, \\
\left\|\partial_{t} \partial_{\theta} v\right\|_{0,0} \leq c \max \left(h_{\theta}^{-r}, h_{t}^{-\frac{r}{2}}\right)\left\|\partial_{t} \partial_{\theta} v\right\|_{-r,-\frac{r}{2},} & 0 \leq r \leq \frac{1}{2} \\
\left\|\left(\partial_{t} \otimes J\right) v\right\|_{0,0} \leq c h_{t}^{-\frac{r}{2}}\left\|\left(\partial_{t} \otimes J\right) v\right\|_{-r,-\frac{r}{2}}, & 0 \leq r<1 .
\end{aligned}
$$

Then the assertion follows from (5.18), (5.19). Let $v \in \mathscr{M}$ and $0 \leq r<1$. By (2.13), (5.1), (5.2),

$$
\begin{aligned}
\|v\|_{r, \frac{r}{2}}^{2} & \leq c \int_{0}^{T}\|v(\cdot, t)\|_{H^{r}}^{2} d t+c \int_{0}^{1}\|v(\theta, \cdot)\|_{H^{\frac{r}{2}}(0, T)}^{2} d \theta \\
& \leq \int_{0}^{T} c h_{\theta}^{-2 r}\|v(\cdot, t)\|_{H^{0}}^{2} d t+\int_{0}^{1} c h_{t}^{-r}\|v(\theta, \cdot)\|_{H^{0}(0, T)}^{2} d \theta \\
& \leq c\left(h_{\theta}^{-2 r}+h_{t}^{-r}\right)\|v\|_{0,0}^{2} \leq c\left(\max \left(h_{\theta}^{-r}, h_{t}^{-\frac{r}{2}}\right)\right)^{2}\|v\|_{0,0}^{2} .
\end{aligned}
$$


We use the duality $H_{00}^{-r,-\frac{r}{2}}\left(\mathbb{R}_{T}\right)=\left(H_{00}^{r, \frac{r}{2}}\left(\mathbb{R}_{T}\right)\right)^{\prime}$ for $0 \leq r<1$. The Schwarz inequality implies

$$
\|v\|_{0,0}^{2} \leq\|v\|_{r, \frac{r}{2}}\|v\|_{-r,-\frac{r}{2}} \leq c \max \left(h_{\theta}^{-r}, h_{t}^{-\frac{r}{2}}\right)\|v\|_{-r,-\frac{r}{2}}\|v\|_{0,0}
$$

which yields (5.17). The estimate (5.18) follows similarly for $0 \leq r<\frac{1}{2}$, since the derivative $\partial_{t} \partial_{\theta} v$ is a piecewise constant function. To see $(5.18)$ for $r=\frac{1}{2}$, we use

$$
\left\|\partial_{t} \partial_{\theta} v\right\|_{0,0} \leq c \max \left(h_{\theta}^{-\frac{1}{4}}, h_{t}^{-\frac{1}{8}}\right)\left\|\partial_{t} \partial_{\theta} v\right\|_{-\frac{1}{4},-\frac{1}{8}}
$$

which further gives

$$
\begin{aligned}
\left\|\partial_{t} \partial_{\theta} v\right\|_{-\frac{1}{4},-\frac{1}{8}}^{2} & \leq\left\|\partial_{t} \partial_{\theta} v\right\|_{0,0}\left\|\partial_{t} \partial_{\theta} v\right\|_{-\frac{1}{2},-\frac{1}{4}} \\
& \leq c \max \left(h_{\theta}^{-\frac{1}{4}}, h_{t}^{-\frac{1}{8}}\right)\left\|\partial_{t} \partial_{\theta} v\right\|_{-\frac{1}{4},-\frac{1}{8}}\left\|\partial_{t} \partial_{\theta} v\right\|_{-\frac{1}{2},-\frac{1}{4}}
\end{aligned}
$$

and therefore

$$
\left\|\partial_{t} \partial_{\theta} v\right\|_{-\frac{1}{4},-\frac{1}{8}} \leq c \max \left(h_{\theta}^{-\frac{1}{4}}, h_{t}^{-\frac{1}{8}}\right)\left\|\partial_{t} \partial_{\theta} v\right\|_{-\frac{1}{2},-\frac{1}{4}} .
$$

Using this with (5.22), we obtain (5.18) with $r=\frac{1}{2}$. The proof of (5.19) is analogous.

Next we consider convergence by using

$$
\|v\|_{0,0}=\left\|\partial_{t} \partial_{\theta} v\right\|_{0,0}+\left\|\left(\partial_{t} \otimes J\right) v\right\|_{0,0}
$$

We have the approximation property

$$
\|\| u-P_{\theta, t} u\left\|_{0,0} \leq c h_{\theta}\right\| \partial_{t} u \|_{2,0}+c h_{t}\left(\|u\|_{0,2}+\left\|\partial_{\theta} u\right\|_{0,2}\right)
$$

for $u \in \mathscr{H}_{00}\left(\mathbb{R}_{T}\right)$ satisfying the assumptions of Lemma 5.1. The proof of this result is a simplified version of the proof of (5.7) and therefore is omitted. Now we obtain

Theorem 5.3. Let the assumptions of Theorem 5.1 be valid. Further, let $c_{0} h \leq$ $h_{\theta} \leq h$ and $c_{0} h^{2} \leq h_{t} \leq c h^{\nu}$, where $h \leq h_{0}$ is sufficiently small and $\nu \geq \frac{6}{5}$. Then we have for the collocation approximation $u_{\Delta}$

$$
\|\| u-u_{\Delta}\|\|_{0,0} \leq \operatorname{ch}\left(\|u\|_{0,2}+\left\|\partial_{\theta} u\right\|_{0,2}+\left\|\partial_{t} u\right\|_{2,0}\right) .
$$

Proof. Because of the lower bounds $c_{0} h \leq h_{\theta}, c_{0} h^{2} \leq h_{t}$ the inverse property (5.16) implies

$$
\begin{aligned}
\| u- & u_{\Delta}\left\|\left.\right|_{0,0} \leq\right\|\left\|u-P_{\theta, t} u\right\|\left\|_{0,0}+\right\|\left\|P_{\theta, t} u-u_{\Delta}\right\|_{0,0} \\
& \leq\|\| u-P_{\theta, t} u\|\|_{0,0}+c h^{-\frac{1}{2}}\left\|\left|P_{\theta, t} u-u_{\Delta} \|\right|_{-\frac{1}{2},-\frac{1}{4}}\right. \\
& \leq\|\| u-P_{\theta, t} u\|\|_{0,0}+c h^{-\frac{1}{2}}\left(\left\|P_{\theta, t} u-u\right\|_{-\frac{1}{2},-\frac{1}{4}}+\left.\left\||| u_{\Delta}-u\right\|\right|_{-\frac{1}{2},-\frac{1}{4}}\right),
\end{aligned}
$$

which together with (5.7), (5.15), and (5.23) yields the assertion (5.24).

Finally, we have the following result for pointwise and $L^{2}$ convergence. 
Theorem 5.4. Under the assumptions of Theorem 5.3 there holds

$$
\begin{array}{r}
\max _{\theta \in[0,1]}\left|\left(u-u_{\Delta}\right)(\theta, t)\right| \leq c t^{\frac{1}{2}} h\left(\|u\|_{0,2}+\left\|\partial_{\theta} u\right\|_{0,2}+\left\|\partial_{t} u\right\|_{2,0}\right), \\
0 \leq t \leq T,
\end{array}
$$

Proof. Since $\left(u-u_{\Delta}\right)(\theta, 0)=0$, we get

$$
\begin{aligned}
\left|\left(u-u_{\Delta}\right)(\theta, t)\right|^{2} & =\left|\int_{0}^{t} \partial_{\tau}\left(u-u_{\Delta}\right)(\theta, \tau) d \tau\right|^{2} \\
& \leq t \int_{0}^{t}\left|\partial_{\tau}\left(u-u_{\Delta}\right)(\theta, \tau)\right|^{2} d \tau .
\end{aligned}
$$

On the other hand, the Sobolev embedding theorem yields

$$
\left|\partial_{\tau}\left(u-u_{\Delta}\right)(\theta, \tau)\right|^{2} \leq c \int_{0}^{1}\left(\left|\partial_{\varphi} \partial_{\tau}\left(u-u_{\Delta}\right)(\varphi, \tau)\right|^{2}+\left|\left(\partial_{\tau} \otimes J\right)\left(u-u_{\Delta}\right)(\tau)\right|^{2}\right) d \varphi \text {. }
$$

Inserting this into (5.27), we obtain

$$
\left|\left(u-u_{\Delta}\right)(\theta, t)\right|^{2} \leq c t\left(\left\|\partial_{\theta} \partial_{t}\left(u-u_{\Delta}\right)\right\|_{0, \mathbb{R}_{T}}^{2}+\left\|\left(\partial_{t} \otimes J\right)\left(u-u_{\Delta}\right)\right\|_{0, \mathbb{R}_{T}}^{2}\right),
$$

which together with (5.24) gives the estimate (5.25). The second assertion follows from (5.25).

\section{NUMERICAL IMPLEMENTATION}

In this section we discuss the numerical solution of the single-layer heat operator equation. For numerical computation we use the basis functions $\psi_{n} \chi_{m}$ of $\mathscr{M}$. Here, $\psi_{n}=\psi_{n}(\theta)$ are the 1 -periodic functions introduced in $\S 2$, and $\chi_{m}=\chi_{m}(t)$ are the Courant functions such that

$$
\chi_{m}\left(t_{l}\right)= \begin{cases}1, & l=m \\ 0, & l \neq m\end{cases}
$$

Writing

$$
u_{\Delta}(\theta, t)=\sum_{n=1}^{N} \sum_{m=1}^{M} \alpha_{m, n} \psi_{n}(\theta) \chi_{m}(t)
$$

we obtain the collocation equations

$$
\begin{aligned}
\left(S u_{\Delta}\right)\left(\theta_{i}, t_{j}\right)=\sum_{n=1}^{N} \sum_{m=1}^{M} \alpha_{m, n}\left(S\left(\psi_{n} \chi_{m}\right)\right)\left(\theta_{i}, t_{j}\right) & =f\left(\theta_{i}, t_{j}\right) \\
& 1 \leq i \leq N, 1 \leq j \leq M .
\end{aligned}
$$

The representation formula (2.2) yields

$$
\left(S\left(\psi_{n} \chi_{m}\right)\right)\left(\theta_{i}, t_{j}\right)=\frac{1}{4 \pi} \int_{0}^{1} \psi_{n}(\varphi) \int_{0}^{t_{j}} \frac{\chi_{m}(\tau)}{\left(t_{j}-\tau\right)} \exp \left(-\frac{\left|\mathbf{x}\left(\theta_{i}\right)-\mathbf{x}(\varphi)\right|^{2}}{4\left(t_{j}-\tau\right)}\right) d \tau d \varphi .
$$

In the direct method (see (1.11), (2.3), (1.6)) we have the right-hand side function

$$
f(\theta, t)=\frac{1}{2} g(\mathbf{x}(\theta), t)+\left(D_{\Gamma} g\right)(\mathbf{x}(\theta), t)=\frac{1}{2} g(\mathbf{x}(\theta), t)+\left(D\left(g\left|\mathbf{x}^{\prime}\right|\right)\right)(\theta, t)
$$


with the double-layer heat operator

$$
(D u)(\theta, t)=\int_{0}^{t} \int_{0}^{1} u(\varphi, \tau) \frac{\mathbf{n}_{\varphi} \cdot(\mathbf{x}(\theta)-\mathbf{x}(\varphi))}{2(t-\tau)} E(\mathbf{x}(\theta)-\mathbf{x}(\varphi), t-\tau) d \varphi d \tau .
$$

We replace the exact values $f\left(\theta_{i}, t_{j}\right)$ by the approximations

$$
\tilde{f}\left(\theta_{i}, t_{j}\right)=\frac{1}{2} g\left(\mathbf{x}\left(\theta_{i}\right), t_{j}\right)+D\left(\widetilde{g\left|\mathbf{x}^{\prime}\right|}\right)\left(\theta_{i}, t_{j}\right),
$$

where $\widetilde{g\left|\mathbf{x}^{\prime}\right|} \in \mathscr{M}$ is the nodal point interpolant of the function $g(\mathbf{x}(\theta), t)\left|\mathbf{x}^{\prime}(\theta)\right|$. Therefore, we need the values

$$
\begin{aligned}
\left(D\left(\psi_{n} \chi_{m}\right)\right)\left(\theta_{i}, t_{j}\right)= & \frac{1}{8 \pi} \int_{0}^{1} \psi_{n}(\varphi) \mathbf{n}_{\varphi} \cdot\left(\mathbf{x}\left(\theta_{i}\right)-\mathbf{x}(\varphi)\right) \\
& \times \int_{0}^{t_{j}} \frac{\chi_{m}(\tau)}{\left(t_{j}-\tau\right)^{2}} \exp \left(-\frac{\left|\mathbf{x}\left(\theta_{i}\right)-\mathbf{x}(\varphi)\right|^{2}}{4\left(t_{j}-\tau\right)}\right) d \tau d \varphi .
\end{aligned}
$$

In (6.1) and (6.2) we abbreviate

$$
\begin{aligned}
& \text { (6.3) } a_{j, m}(i, \varphi)=\int_{0}^{t_{j}} \frac{\chi_{m}(\tau)}{\left(t_{j}-\tau\right)} \exp \left(-\frac{\left|\mathbf{x}\left(\theta_{i}\right)-\mathbf{x}(\varphi)\right|^{2}}{4\left(t_{j}-\tau\right)}\right) d \tau \\
& \text { (6.4) } b_{j, m}(i, \varphi)=\left|\mathbf{x}\left(\theta_{i}\right)-\mathbf{x}(\varphi)\right|^{2} \int_{0}^{t_{j}} \frac{\chi_{m}(\tau)}{\left(t_{j}-\tau\right)^{2}} \exp \left(-\frac{\left|\mathbf{x}\left(\theta_{i}\right)-\mathbf{x}(\varphi)\right|^{2}}{4\left(t_{j}-\tau\right)}\right) d \tau
\end{aligned}
$$

and define the $N \times N$ square matrices $A_{j, m}, B_{j, m}, 1 \leq j, m \leq M$, by

$$
\begin{aligned}
& \left(A_{j, m}\right)_{i, n}=\frac{1}{4 \pi} \int_{0}^{1} \psi_{n}(\varphi) a_{j, m}(i, \varphi) d \varphi, \quad 1 \leq i, n \leq N, \\
& \left(B_{j, m}\right)_{i, n}=\frac{1}{8 \pi} \int_{0}^{1} \psi_{n}(\varphi) \frac{\mathbf{n}_{\varphi} \cdot\left(\mathbf{x}\left(\theta_{i}\right)-\mathbf{x}(\varphi)\right)}{\left|\mathbf{x}\left(\theta_{i}\right)-\mathbf{x}(\varphi)\right|^{2}} b_{j, m}(i, \varphi) d \varphi,
\end{aligned}
$$

$$
1 \leq i, n \leq N
$$

From (6.3), (6.4) there follows $A_{j, m}=B_{j, m}=0, j<m$, and moreover,

$$
A_{j, m}=A_{j-m+1,1}=: A_{j-m+1}, \quad B_{j, m}=B_{j-m+1,1}=: B_{j-m+1}, \quad j \geq m,
$$

which yield the block lower triangular matrices $\mathscr{A}=\left(A_{j, m}\right), \mathscr{B}=\left(B_{j, m}\right)$,

$$
\mathscr{A}=\left(\begin{array}{cccc}
A_{1} & 0 & \ldots & 0 \\
A_{2} & A_{1} & \ldots & 0 \\
\vdots & \vdots & \ddots & 0 \\
A_{m} & \ldots & \ldots & A_{1}
\end{array}\right), \quad \mathscr{B}=\left(\begin{array}{cccc}
B_{1} & 0 & \ldots & 0 \\
B_{2} & B_{1} & \ldots & 0 \\
\vdots & \vdots & \ddots & 0 \\
B_{m} & \ldots & \ldots & B_{1}
\end{array}\right) .
$$

Introducing the $N$-dimensional vectors

$$
\alpha_{m}=\left(\begin{array}{c}
\alpha_{m, 1} \\
\vdots \\
\alpha_{m, N}
\end{array}\right), \quad G_{m}=\left(\begin{array}{c}
g\left(\mathbf{x}\left(\theta_{1}\right), t_{m}\right) \\
\vdots \\
g\left(\mathbf{x}\left(\theta_{N}\right), t_{m}\right)
\end{array}\right), \quad \tilde{G}_{m}=\left(\begin{array}{c}
g\left(\mathbf{x}\left(\theta_{1}\right), t_{m}\right)\left|\mathbf{x}^{\prime}\left(\theta_{1}\right)\right| \\
\vdots \\
g\left(\mathbf{x}\left(\theta_{N}\right), t_{m}\right)\left|\mathbf{x}^{\prime}\left(\theta_{N}\right)\right|
\end{array}\right),
$$

we have for the direct method the right-hand side

$$
\tilde{\mathscr{F}}=\left(\begin{array}{c}
\tilde{F}_{1} \\
\tilde{F}_{2} \\
\vdots \\
\tilde{F}_{m}
\end{array}\right)=\frac{1}{2}\left(\begin{array}{c}
G_{1} \\
G_{2} \\
\vdots \\
G_{m}
\end{array}\right)+\left(\begin{array}{cccc}
B_{1} & 0 & \ldots & 0 \\
B_{2} & B_{1} & \ldots & 0 \\
\vdots & \vdots & \ddots & 0 \\
B_{m} & B_{m-1} & \ldots & B_{1}
\end{array}\right)\left(\begin{array}{c}
\tilde{G}_{1} \\
\tilde{G}_{2} \\
\vdots \\
\tilde{G}_{m}
\end{array}\right) .
$$


This gives the linear equations $\mathscr{A} \alpha=\tilde{\mathscr{F}}$, where the components $\alpha_{i}$ are obtained by applying the formulas

$$
A_{1} \alpha_{1}=\tilde{F}_{1}, \quad A_{1} \alpha_{k}=\tilde{F}_{k}-\sum_{\nu=1}^{k-1} A_{k+1-\nu} \alpha_{\nu}, \quad k=2, \ldots, M .
$$

Because of the coercivity of the single-layer operator, the matrix $\mathscr{A}$, or equivalently, $A_{1}$ is invertible. In the computations we have used the Gaussian algorithm for inversion of the matrix $A_{1}$.

Let us turn to the evaluation of the matrix elements. We compute the double integrals in the matrix elements by using analytical integration with respect to the time-variable. For this we introduce the exponential integral function [1]

$$
E_{1}(z)=\int_{z}^{\infty} \frac{e^{-z}}{z} d z
$$

which has the logarithmic singularity ( $\gamma$ is Euler's constant)

$$
E_{1}(z)=-\gamma-\log (z)-\sum_{k=1}^{\infty} \frac{(-1)^{k}}{k k !} z^{k}, \quad|\arg z|<\pi,
$$

at the origin $z=0$. When $z$ is approaching infinity, the function $E_{1}(z)$ decays exponentially. Denoting

$$
f_{s}(z)=E_{1}(z)+z E_{1}(z)-e^{-z}, \quad f_{d}(z)=z E_{1}(z)-e^{-z},
$$

we obtain

$$
\begin{aligned}
a_{j, m}(i, \varphi)= & (j-m+1) f_{s}\left(\frac{\left|\mathbf{x}\left(\theta_{i}\right)-\mathbf{x}(\varphi)\right|^{2}}{4(j-m+1) h_{t}}\right)-2(j-m) f_{s}\left(\frac{\left|\mathbf{x}\left(\theta_{i}\right)-\mathbf{x}(\varphi)\right|^{2}}{4(j-m) h_{t}}\right) \\
& +(j-m-1) f_{s}\left(\frac{\left|\mathbf{x}\left(\theta_{i}\right)-\mathbf{x}(\varphi)\right|^{2}}{4(j-m-1) h_{t}}\right), \quad 1 \leq m \leq j-2,
\end{aligned}
$$

and

$$
\begin{aligned}
& a_{j, m}(i, \varphi)=2 f_{s}\left(\frac{\left|\mathbf{x}\left(\theta_{i}\right)-\mathbf{x}(\varphi)\right|^{2}}{8 h_{t}}\right)-2 f_{s}\left(\frac{\left|\mathbf{x}\left(\theta_{i}\right)-\mathbf{x}(\varphi)\right|^{2}}{4 h_{t}}\right), \quad m=j-1, \\
& a_{j, m}(i, \varphi)=f_{s}\left(\frac{\left|\mathbf{x}\left(\theta_{i}\right)-\mathbf{x}(\varphi)\right|^{2}}{4 h_{t}}\right), \quad m=j .
\end{aligned}
$$

For $b_{j, m}(i, \varphi)$ we have similar expressions with the function $f_{s}$ replaced by $f_{d}$.

With respect to the space-variable, numerical integration has to be applied. We decompose the integrand in (6.5) into a singular part with logarithmic singularity and a smooth part. Exact integration is used for the singular part. For the smooth part, Gaussian quadrature with three integration points in the reference interval is applied. This method is analogous to the computations for the harmonic single-layer operator $[12,13]$. We remark that the integrand in (6.6) is a continuous function but the first derivatives have a logarithmic singularity. This fact has been taken into account in the numerical integration.

\section{NuMERICAL RESULTS}

In the following examples the spatial domain is the unit circle $\Omega=\{\mathbf{x}|| \mathbf{x} \mid \leq$ 1) with boundary $\Gamma$. We use the direct boundary integral approach, which leads 
to the single-layer operator equation $S u=f$. For the solution we have $u=$ $\left|\mathbf{x}^{\prime}\right| \partial_{n} \Phi$, where $\partial_{n} \Phi$ is equal to the interior limit of the normal derivative. The examples are taken from [19], where the Galerkin approximation is considered. For other results see $[21,25]$.

First we consider the heat conduction problem

$$
\begin{cases}\partial_{t} \Phi-\Delta \Phi=0 & \text { in } \Omega \times(0,1) \\ \left.\Phi\right|_{\Sigma_{T}}=t^{2} & \text { on } \Gamma \times(0,1) \\ \Phi(\mathbf{x}, 0)=0, & \mathbf{x} \in \Omega\end{cases}
$$

which has the solution

$$
\Phi(r, \theta, t)=t^{2}-4 \sum_{k=1}^{\infty} \frac{J_{0}\left(\beta_{0, k} r\right)}{\beta_{0, k}^{3} J_{1}\left(\beta_{0, k}\right)}\left[t-\frac{\left(1-e^{\left.-\beta_{0, k}^{2}\right)}\right.}{\beta_{0, k}^{2}}\right],
$$

with the normal derivative

$$
\partial_{n} \Phi(\theta, t)=t-4 \sum_{k=1}^{\infty} \frac{\left(1-e^{-\beta_{0, k}^{2} t}\right)}{\beta_{0, k}^{4}} .
$$

In the formulas (7.2), (7.3), $J_{0}(z)$ and $J_{1}(z)$ are Bessel functions and the numbers $\beta_{0, k}$ are the positive zeros of $J_{0}(z)$. In problem (7.1), the boundary condition and the exact normal derivative are independent of the space variable. The same property is also satisfied by the collocation approximation. We have calculated the numerical solution to the single-layer operator equation. The corresponding $L^{2}$ errors with 8, 16, 32, 64 nodes on the spatial boundary, and $8,16,32,64,128$ nodes on the time boundary $[0,1]$, are presented in Table 1 . The results indicate that convergence is slightly faster than quadratic with respect to the time-step. The rate is computed by using the formula $\ln (e(64) / e(128)) / \ln 2$, where $e(128), e(64)$ are the $L^{2}$ errors for $h_{t}=\frac{1}{128}, \frac{1}{64}$, respectively.

TABLE 1. Absolute $L^{2}$-errors

$\begin{array}{ccccccc} & 8 & 16 & 32 & 64 & 128 & \text { rate } \\ 8 & 0.0003920 & 0.0000750 & 0.0000148 & 0.0000033 & 0.00000113 & 1.5 \\ 16 & 0.0002761 & 0.0000523 & 0.0000099 & 0.0000018 & 0.00000037 & 2.3 \\ 32 & 0.0001952 & 0.0000369 & 0.0000069 & 0.0000019 & 0.00000024 & 2.4 \\ 64 & 0.0001380 & 0.0000261 & 0.0000049 & 0.0000009 & 0.00000017 & 2.4\end{array}$

In the second example,

$$
\begin{cases}\partial_{t} \Phi-\Delta \Phi=0 & \text { in } \Omega \times(0,1) \\ \left.\Phi\right|_{\Sigma_{T}}=t^{2} \cos (2 \pi \theta) & \text { on } \Gamma \times(0,1) \\ \Phi(\mathbf{x}, 0)=0, & \mathbf{x} \in \Omega\end{cases}
$$

the given boundary data depends on both variables $\theta$ and $t$. The solution

$$
\Phi(r, \theta, t)=\left\{r t^{2}-4 \sum_{k=1}^{\infty} \frac{J_{1}\left(\beta_{1, k} r\right)}{\beta_{1, k}^{3} J_{2}\left(\beta_{1, k}\right)}\left[t-\frac{\left(1-e^{\left.-\beta_{1, k}^{2}\right)}\right.}{\beta_{1, k}^{2}}\right]\right\} \cos (2 \pi \theta)
$$


has the normal derivative

$$
\partial_{n} \Phi(\theta, t)=\left\{t^{2}+0.5 t-4 \sum_{k=1}^{\infty} \frac{\left(1-e^{-\beta_{1, k}^{2} t}\right)}{\beta_{1, k}^{4}}\right\} \cos (2 \pi \theta)
$$

where the numbers $\beta_{1, k}$ are the positive roots of the function $J_{1}(z)$. In Table 2 we have listed the $L^{2}$ errors with $8,16,32,64$ nodes on the spatial boundary, and $4,8,16,32,64,128$ nodes on the time boundary $[0,1]$.

TABLE 2. Absolute $L^{2}$ errors

$\begin{array}{ccccccc} & 4 & 8 & 16 & 32 & 64 & 128 \\ 8 & 0.003615 & 0.003077 & 0.002339 & 0.001700 & 0.001216 & 0.000864 \\ 16 & 0.000935 & 0.000518 & 0.000426 & 0.000319 & 0.000230 & 0.000164 \\ 32 & 0.000742 & 0.000134 & 0.000070 & 0.000056 & 0.000042 & 0.000030 \\ 64 & 0.000567 & 0.000105 & 0.000018 & 0.000009 & 0.000007 & 0.000005\end{array}$

For this example we illustrate the $L^{2}$ convergence in Figure 1. The curves corresponding to constant space steps $h_{\theta}=\frac{1}{8}, \frac{1}{16}, \frac{1}{32}, \frac{1}{64}$ are drawn by using a logarithmic scale. Thus, the slope represents the rate of the convergence. The lines starting from the upper left corner correspond to convergence rates $\mathscr{O}(h), \mathscr{O}\left(h^{\frac{3}{2}}\right), \mathscr{O}\left(h^{2}\right)$. It seems that convergence is quadratic with respect to the $L^{2}$ norm if the time-step is sufficiently small compared to the space-step.

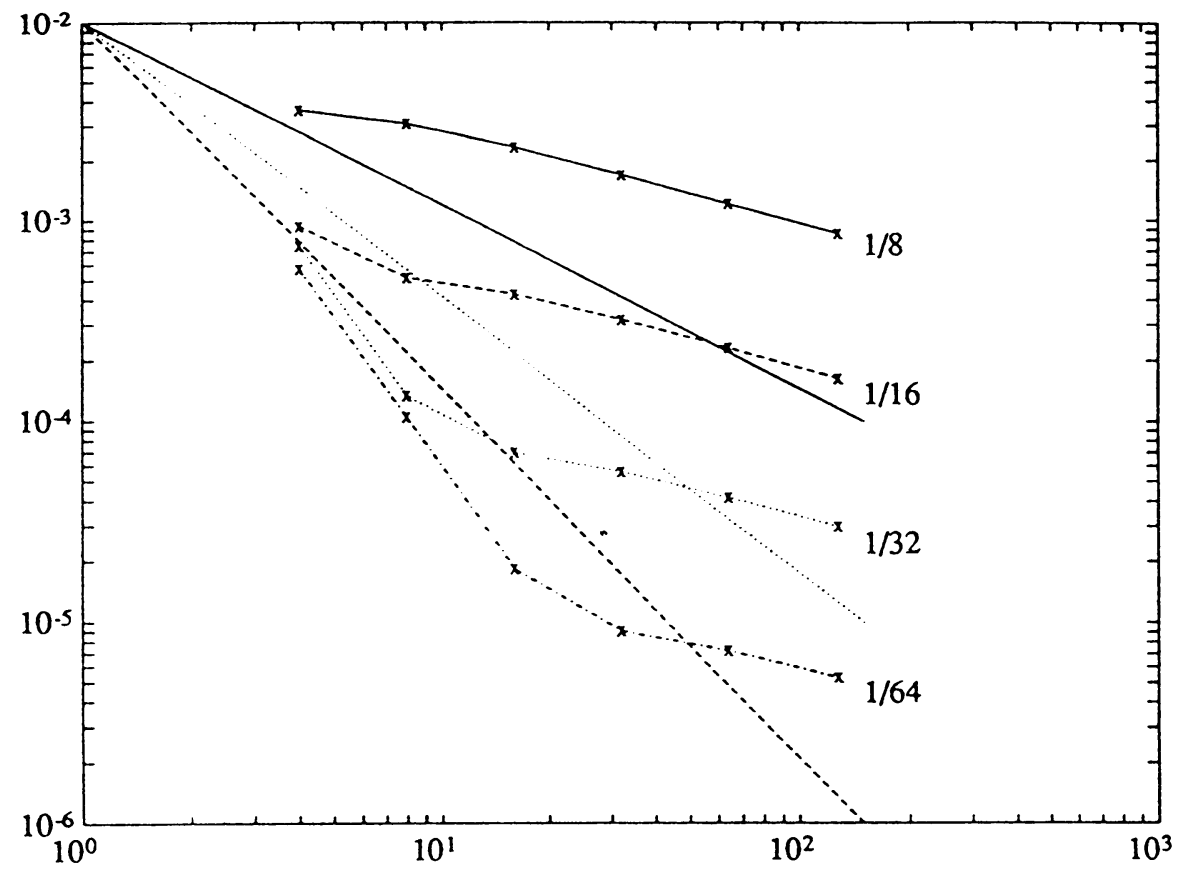

FIGURE 1 
We have also done experiments regarding the run time of the program. In Table 3 the percentile distribution of execution time is presented when the program, written in Turbo C language, was run on a Everex Step $386 \mathrm{SX} / 387$ $\mathrm{SX}, 16 \mathrm{MHz}$. The data for Table 3 was collected from the output of the Turbo Profiler program.

TABLE 3. Run time data for the program

$\begin{array}{lccc}\text { space/time meshes } & 16 / 16 & 32 / 16 & 16 / 32 \\ \text { double-layer operator } & 23 & 24 & 19 \\ \text { single-layer operator } & 20 & 22 & 17 \\ \text { exponential integral } & 20 & 22 & 16 \\ \text { other functions } & 04 & 02 & 04 \\ \text { matrix multiplication } & 20 & 11 & 33 \\ \text { matrix inversion } & 02 & 09 & 01 \\ \text { others } & 11 & 10 & 10 \\ \text { total execution time } & 37 \mathrm{sec} & 71 \mathrm{sec} & 85 \mathrm{sec}\end{array}$

It turns out that about $56-70 \%$ of the execution time is used for setting up the linear equations. We remark that special care must be taken in the computation of the exponential integral function.

\section{BIBLIOGRAPHY}

1. M. Abramowitz and I. A. Stegun (eds.), Handbook of mathematical functions with formulas, graphs and mathematical tables, U.S. Government Printing Office, Washington, DC, 1971.

2. D. N. Arnold and J. Saranen, On the asymptotic convergence of spline collocation methods for partial differential equations, SIAM J. Numer. Anal. 21 (1984), 459-472.

3. D. N. Arnold and W. L. Wendland, On the asymptotic convergence of collocation methods, Math. Comp. 41 (1983), 349-381.

4. I. Babuška and A. K. Aziz, Survey lectures on the mathematical foundations of the finite element method, The Mathematical Foundations of the Finite Element Method with Applications to Partial Differential Equations (A. K. Aziz, ed.), Academic Press, New York, 1972, pp. 3-359.

5. A. Bamberger and Duong T. Ha, Formulation variationnelle espace-temps pour le calcul par potentiel retardé de la diffraction d'une onde acoustique (I), Math. Methods Appl. Sci. 8 (1986), 405-435.

6. Formulation variationnelle pour le calcul de la diffraction d'une onde acoustique par une surface rigide (II), Math. Methods Appl. Sci. 8 (1986), 598-608.

7. M. Costabel, Boundary integral operators for the heat equation, Integral Equations Operator Theory 13(4) (1990), 498-552.

8. M. Costabel, K. Onishi, and W. Wendland, A boundary element collocation method for the Neumann problem of the heat equation, Inverse and Ill-Posed Problems $(\mathrm{H}$. W. Engl and C. W. Groetsch, eds.), Academic Press, New York, 1987, pp. 369-384.

9. J. Elschner and G. Schmidt, On spline interpolation in periodic Sobolev spaces, Preprint 01/83, Dept. Math. Akademie der Wissenschaften der DDR, Berlin, 1985.

10. A. Friedman, Partial differential equations of parabolic type, Robert E. Krieger Publishing Co., Malabar, FL, 1983.

11. M. Hamina and J. Saranen, On the collocation approximation for the single layer heat operator equation, Z. Angew. Math. Mech. 71 (1991), 629-631. 
12. G. C. Hsiao, P. Kopp, and W. L. Wendland, A Galerkin collocation method for some integral equations of the first kind, Computing 25 (1980), 89-130.

13. Some applications of a Galerkin-collocation method for boundary integral equations of the first kind, Math. Methods Appl. Sci. 6 (1984), 280-325.

14. G. C. Hsiao and J. Saranen, Coercivity of the single layer heat operator, Technical Report 89-2, Department of Mathematical Sciences, University of Delaware, 1989.

15. _ Boundary integral solution of some heat conduction problems, Proc. Internat. Conf. on Integral Equations and Inverse Problems, Varna, September 18-23, 1989.

16. _ Boundary integral solution of the two-dimensional heat equation, Math. Methods Appl. Sci. 16 (1993), 87-114.

17. J. L. Lions and E. Magenes, Non-homogeneous boundary value problems and applications I, Springer-Verlag, Berlin, Heidelberg, New York, 1972.

18. _ Non-homogeneous boundary value problems and applications II, Springer-Verlag, Berlin, Heidelberg, New York, 1972.

19. J. P. Noon, The single layer heat potential and Galerkin boundary element methods for the heat equation, Ph.D. Thesis, University of Maryland, 1988.

20. K. Onishi, Convergence in the boundary element method for the heat equation, TRU Math. 17 (1981), 213-225.

21. H. G. L. Pina and J. L. M. Fernandes, Applications in transient heat conduction, Topics in Boundary Element Research 1 (C. A. Brebbia, ed.), Springer-Verlag, New York, 1972, pp. 41-58.

22. W. Pogorzelski, Integral equations and their applications, Pergamon Press, Oxford, 1966.

23. J. Saranen, The modified quadrature method for logarithmic-kernel integral equations on closed curves, J. Integral Equations Appl. 3 (1991), 575-600.

24. J. Saranen and I. H. Sloan, Quadrature methods for logarithmic-kernel integral equations on closed curves, IMA J. Numer. Anal. 12 (1992), 167-187.

25. F. Sgallari, $A$ weak formulation of boundary integral equations for time dependent problems, Appl. Math. Modelling 9 (1985), 295-301.

Section of Mathematics, Faculty of Technology, University of Oulu, SF-90570 OULu, FinLAND 